\title{
SORET AND DUFOUR EFFECTS ON MHD RADIATIVE HEAT AND MASS TRANSFER FLOW OF A SISKO FLUID FLOW OVER A STRETCHING SHEET WITH NON-UNIFORM HEAT SOURCE/SINK
}

\author{
S. SUDHAKAR REDDY ${ }^{*}$, G. RAVI SANKAR ${ }^{2} \&$ M. KRISHNA MURTHY ${ }^{3}$ \\ ${ }^{1 *}$ Dept of Mathematics, Sri Venkateswara Engineering College, Tirupati, A.P., India \\ ${ }^{2}$ Dept of Mathematics, Sri Venkateswara College of Engineering, Kadapa, A.P., India
}

${ }^{3}$ Dept of Mathematics, School of Applied Sciences, REVA University, Bengaluru, Karnataka, India

\begin{abstract}
The present analysis Soret and Dufour effects on MHD radiative heat and mass transfer flow of a Sisko fluid flow over a stretching sheet with non-uniform heat source/sinkare studied. The governing partial differential equations are transformed into ordinary differential equations with the help of similarity transformation. The nonlinear differential equations are solved by using shooting technique along with Fourth order Runge-Kutta method. The effect of pertinent parameters on the fluid velocity, the temperature and the concentration are shown in graphically. While the Shear stress, the Nusselt number and Sherwood number are shown in tabular form.

KEYWORDS: MHD; Shooting Method; Sisko Fluid; Stretching Sheet
\end{abstract}

Received: Jun 08, 2020; Accepted: Jun 28, 2020; Published: Sep 12, 2020; Paper Id.: IJMPERDJUN20201175

\section{NOMENCLATURE}

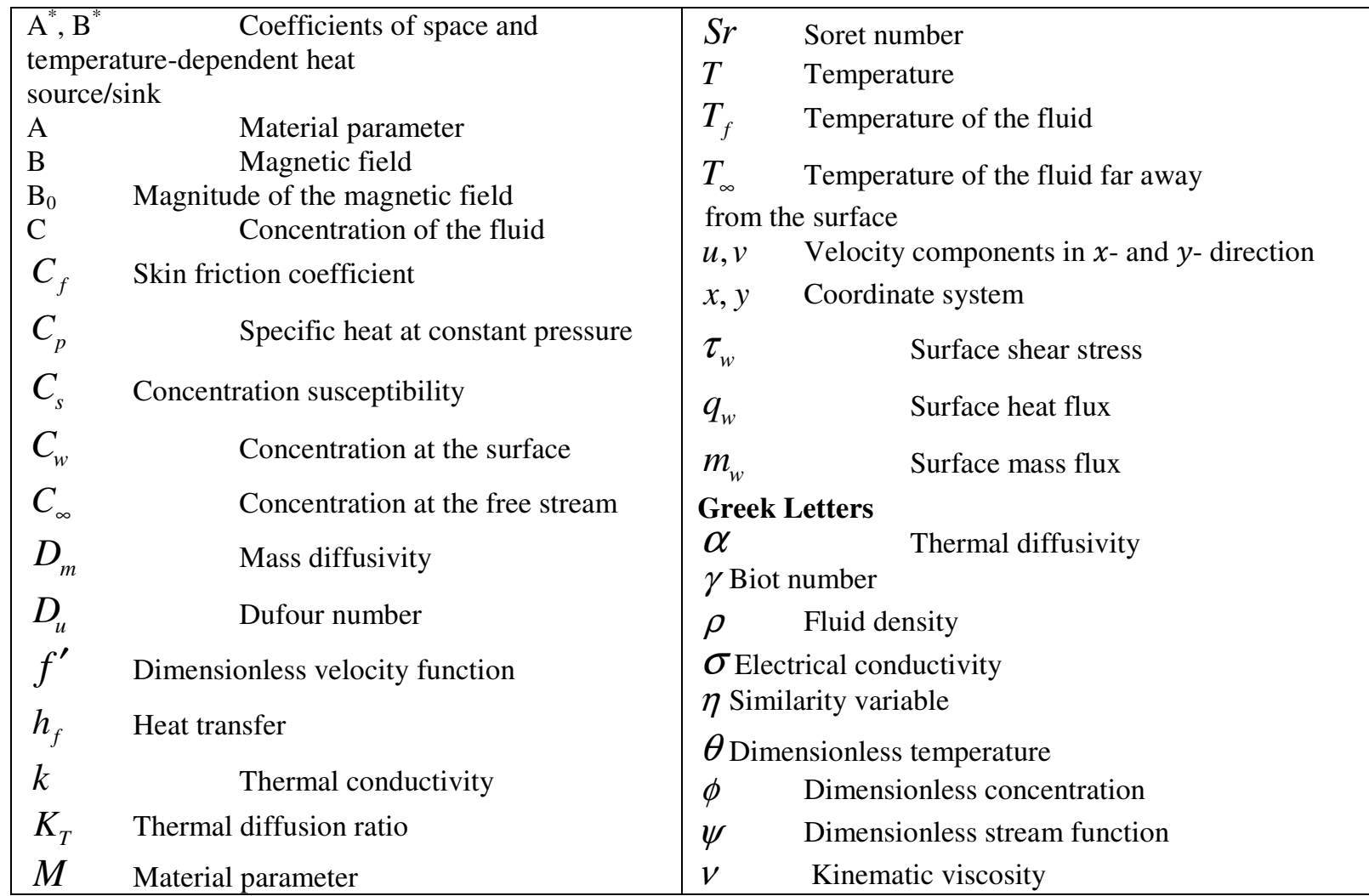




\begin{tabular}{|ll|l|}
\hline$m_{w}$ & Surface mass flux & $\mu$ Coefficient of fluid viscosity [pas] \\
$n$ & Power law index & Subscripts \\
$N u_{x}$ & Nusselt number & $f \quad$ Condition at wall \\
$\mathrm{Pr}$ & Prandtl number & $\infty \quad$ Condition at fluid \\
$q_{w}$ & Surface heat flux & Condition at infinity \\
$S$ & Stretching parameter & \multicolumn{2}{|}{} \\
$S c$ & Schmidt number & \\
$S h_{x}$ & Sherwood number & \\
\hline
\end{tabular}

\section{INTRODUCTION}

The analysis of non-Newtonian fluids has gained an abundance of attention by the researchers from the last few decades due to their wider applications in industries. So many non-Newtonianmodels were suggested by the earlier researchers to study the physical properties of these fluids. Among them, the power law fluid model is most felicitous model to presage the attitude of non-Newtonian fluids. But it can predict fluid properties in the power law region only and hence fails to analyze flow characteristics when the shear rate becomes very small or large. To surmount this difficulty Sisko [32] proposed a model, which predicts fluid properties in a power law region and also at high shear rate. Moreover experimentally it is verified that many realistic fluids such as waterborne coatings, drilling muds, biological fluids, cement slurries, paints etc. follow Sisko fluid model. A broad description of the non-Newtonian fluid behavior in both steady and unsteady flow is elucidated by Cross [8] and Chhabbra and Richardson [7]. Afterwards, Malik et al. [16] presented the series solution for MHD Sisko fluid flow over a stretching cylinder. Awais et al. [4] explored the magnetohydrodynamic flow of Sisko fluid near the axisymmetric stagnation point towards a stretching cylinder. Khan et al. [13] described the magnetohydrodynamic flow of Sisko fluid in annular pipe and calculated the numerical solution of the problem. They concluded that the velocity of Sisko fluid is much less than Newtonian fluid. Also, a few useful investigations on Sisko fluid have been reported in [31,19,9]. Hayat et al. [11] studied the boundary layer flow of Jeffrey fluid with convective boundary conditions. Munir et al. [21] studied the forced convective heat transfer inboundary layer flow of Sisko fluid over a nonlinear stretching sheet. It is worth pointing out that few investigations on the Sisko fluid with heat transfer have been studied by Malik et al. [17] and Khan et al. [14].

The effect of heat source/sink on heat transfer is another significant aspect in view of many physical problems. Heat generation or absorption may change the heat distribution in the fluid which consequently affects the particle deposition rate in the system such as semiconductors, electronic devices and nuclear reactors. The heat source/sink effects in thermal convection are considerable where there may exist high temperature differences between the surface and the ambient fluid. Tsai et al. [33] examined the unsteady flow over a stretching surface with non-uniform heat source/sink. The unsteady boundary layer flow over a moving permeable stretching surface with non-uniform heat source/sink and thermal radiation is studied by Pal [24]. Also, Pal [25] studied the effects of thermal radiation and non-uniform heat source/sink on mixed convection flow over an unsteady stretching sheet. He concluded that non-uniform heat source/sink parameters enhance the fluid velocity. The radiative flow of couple stress fluid over a stretched cylinder in the presence of nonuniform heat source/sink was studied by Hayat et al. [10]. Reddy et al. [30] studied the flow of dusty nanofluid over a cone with non-uniform heat source/sink. The MHD mixed convection flow of a radiative nanofluid over an inclined stretching sheet with non-uniform heat source/sink was considered by Mehmood et al. [18]. Raju et al. [28] analyzed the water based 
nanofluid flow over an unsteady permeable stretching sheet in the presence of non-uniform heat source/sink and chemical reaction. Monica and Sucharitha [20] investigated the hydrodynamic stagnation point flow of a Casson fluid over a nonlinear stretching sheet with slip conditions, thermal radiation and non-uniform heat source/sink. The influence of nonuniform heat source/sink on ethylene glycol nanofluid flow over a stretching sheet by using the Jeffrey fluid model is analyzed by Avinash et al. [3]. They concluded that the non-uniform heat source/sink parameters acts as heat generators.

In the recent years convective heat and mass transfer over a stretching sheet plays a major role because of its tremendous applications in engineering and sciences. For this reason many researchers are focusing in this area. Convective heat and mass transfer with chemical reaction plays a vital role in meteorological phenomena, burning of haystacks, spray drying of milk, fluidized bed catalysis and cooling towers. Bhuvaneswari et al. [6] examined the flow, heat and mass transfer of an incompressible viscous fluid past a semi-infiniteinclined surface with first-order homogeneous chemical reaction. Raju and Sandeep [27] investigated the heat and mass transfer characteristics of Newtonian and nonNewtonian fluids by considering the cross diffusion effect.Usually, when heat and mass transfer takes place at the same time, the energy flux can be provided by both concentration and temperature gradients. The energy flux caused by concentration gradients is called diffusion thermo or Dufour effect and energy flux induced by temperature gradients is called thermal diffusion or Soret effect. In most of the studies with heat and mass transfer, Soret and Dufour effects are neglected on the basis that they are of small order of magnitude than the effects described by Fourier's and Fick's law of diffusion. But these effects are considered as second order phenomena and may be considerable in areas such as hydrology, petrology, geosciences etc.

In all the above studies, the Soret/Dufour effects are assumed to be insignificant. But, such effects are considerable when density differences exist in the flow regime. In view of this, Mabood and Ibrahim [15] examined the MHD mixed convection flow of a micropolar fluid over a stretching sheet through porous medium with the effects of nonuniform heat source/sink, thermal radiation and thermo-diffusion. The MHD mixed convective unsteady stretching sheet flow in the presence of non-uniform heat source/sink, thermo-diffusion,chemical reaction and viscous dissipation is analyzed by Bhukta et al. [5]. Hayat et al. [12] discussed the cross diffusion effects (Soret and Dufour) on mixed convection flow of a viscoelastic fluid over a vertical stretching surface. Very recently, Reddy et al. [29] studied the effect of Soret and Dufour numbers on magnetohydrodynamic flow of fluids over three different geometries using CattaneoChristov heat flux model. The free convection flow of a Casson fluid flow over a vertical cone through porous medium with non- uniform heat source/sink, diffusion thermo and thermo diffusion and chemical reaction is considered by Mythili et al. [22]. They concluded that the velocity profiles are enhanced by increasing cross diffusion parameters. Alam and Rahman [2] studied the Soret and Dufour effects on mixed convection flow with variable suction. Numerical investigations of heat and mass transfer in non-Newtonian fluids over various geometries were discussed by the researchers $[1,26]$. In this paper, we will discuss the effect of non-uniform heat source/sink on MHD flow of a Sisko fluid over a surface with cross diffusion effect.

objective of the present paper is to study the impact of cross diffusion and non uniform heat source/sink on Sisko fluid flow over a non-linear stretching sheet in the presence of uniform transverse magnetic field. The convective type boundary condition of temperature is considered. The set of dimensional partial differential equations is transformed into dimensionless ordinary differential equations by using suitable similarity transformations and then solved numerically by using the MATLAB bvp4c package. The obtained results are presented and discussed through graphs and tables. 


\section{MATHEMATICAL FORMULATION}

We consider steady, laminar, two-dimensional and hydro magnetic boundary layer flow of an incompressible, viscous and electrically conducting non-Newtonian fluid (Sisko fluid) over an isothermal stretching sheet. The effects of Soret and Dufour numbers are taken into account. The Dufour effect may be described by a second-order concentration derivative w.r.t the transverse coordinates in the energy equation where as the Soret effect is described by the second-order temperature derivative in the mass diffusion equation.

It is assumed that the flow is caused due to stretching of the sheet. The flow direction is along $\mathrm{x}$-axis and $\mathrm{y}$-axis is normal to it. The flow takes place at $y \geq 0$ (see Fig.1). The temperature and concentration at the surface are in the form $k \frac{\partial T(x, 0)}{\partial y}=-h_{f}\left[T_{f}-T(x, 0)\right]$ and $C=C_{w}$ respectively. The temperature and concentration far away the surfaces are $T_{\infty}$ and $C_{\infty}$ respectively. The sheet is stretching with velocity $U(x)=c x^{s}$, where c and s are non-negative real numbers. A uniform transverse magnetic field $B_{0}$ is applied normal to the flow direction. The magnetic Reynolds number is assumed to be very small. So, the induced magnetic field is neglected. The convective boundary condition on temperature field is considered.

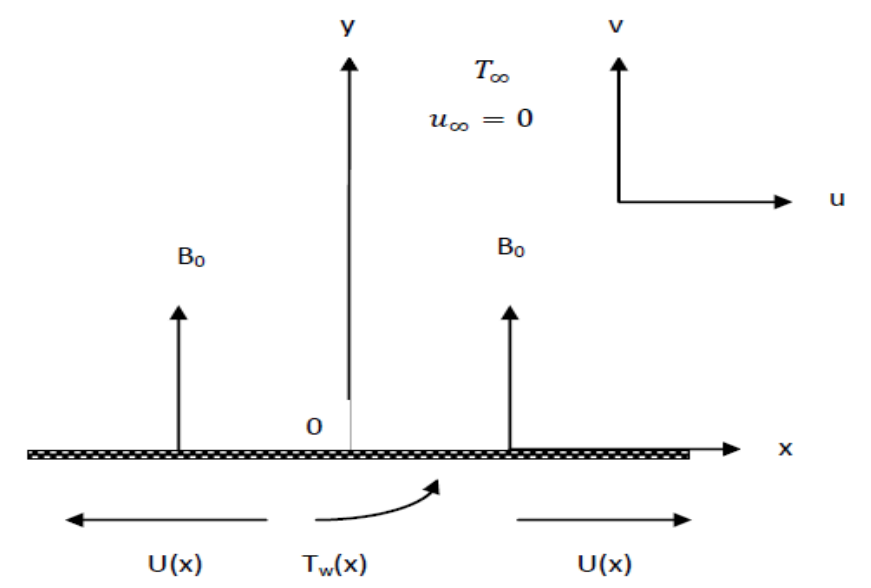

Figure 1: Schematic diagram of the problem

The governing equations for two-dimensional boundary layer flow corresponding to the velocity, temperature and concentration are

$$
\begin{aligned}
& \frac{\partial u}{\partial x}+\frac{\partial v}{\partial y}=0 \\
& u \frac{\partial u}{\partial x}+v \frac{\partial u}{\partial y}=\frac{a}{\rho} \frac{\partial^{2} u}{\partial y^{2}}-\frac{b}{\rho} \frac{\partial}{\partial y}\left(-\frac{\partial u}{\partial y}\right)^{n}-\frac{\sigma B_{0}^{2}}{\rho} u \\
& u \frac{\partial T}{\partial x}+v \frac{\partial T}{\partial y}=\alpha \frac{\partial^{2} T}{\partial y^{2}}+\frac{q^{\prime \prime \prime}}{\rho C_{p}}+\frac{D_{m} K_{T}}{C_{s} C_{p}} \frac{\partial^{2} C}{\partial y^{2}}
\end{aligned}
$$




$$
u \frac{\partial C}{\partial x}+v \frac{\partial C}{\partial y}=D_{m} \frac{\partial^{2} C}{\partial y^{2}}+\frac{D_{m} K_{T}}{T_{m}} \frac{\partial^{2} T}{\partial y^{2}}
$$

with the boundary conditions

$$
\begin{aligned}
& u(x, y)=U=c x^{s}, v(x, y)=0, C=C_{w}, k \frac{\partial T(x, 0)}{\partial y}=-h_{f}\left[T_{f}-T(x, 0)\right] \text { at } y=0 \\
& u \rightarrow 0, T \rightarrow T_{\infty}, C \rightarrow C_{\infty} \text { as } y \rightarrow \infty
\end{aligned}
$$

In equations (1)-(4), $a, b$ and $n(\geq 0)$ are the mathematical constants. $u$ and $v$ are the velocity components along $x-$ and $y$ - directions respectively. $T_{f}$ is the temperature of the fluid below the plate, $h_{f}$ the heat transfer coefficient, $T, C$ are temperature and concentration of the fluid respectively.

In equations (2)-(4), $\alpha=\frac{k}{\rho C_{p}}$ the thermal diffusivity, $\sigma$ is the electrical conductivity of the fluid, $\rho$ is the fluid density, $k$ is the thermal conductivity, $B_{0}$ is the magnitude of applied magnetic field, $C_{w}$ is the species concentration at the plate surface and $C_{p}$ is the specific heat at constant pressure, $T_{m}$ is the mean fluid temperature, $K_{T}$ is the thermal diffusion ratio and $C_{s}$ is the concentration susceptibility. The non-uniform heat source/sink $q^{\prime \prime \prime}$ is given by $q^{\prime \prime \prime}=\frac{k U_{w}(x)}{x \nu}\left[A *\left(T_{f}-T_{\infty}\right) f^{\prime}(\eta)+B *\left(T-T_{\infty}\right)\right]$, where $A^{*}$ and $B^{*}$ are the coefficients of space and temperature-dependent heat source/sink, respectively. The case $A^{*}>0, B^{*}>0$ corresponds to internal heat generation while $A^{*}<0, B^{*}<0$ corresponds to internal heat absorption.

\section{SOLUTION OF THE PROBLEM}

The suitable similarity transformations are defined as follows

$$
\begin{aligned}
& u(x, y)=U f^{\prime}(\eta), v(x, y)=-U \operatorname{Re}_{b}^{\frac{-1}{n+1}} \frac{1}{n+1}\left[\{s(2 n-1)+1\} f(\eta)+\{s(2-n)-1\} \eta f^{\prime}(\eta)\right] \\
& \eta=\frac{y}{x} \operatorname{Re}_{b}^{\frac{1}{n+1}}, \psi=U x \operatorname{Re}_{b}^{\frac{-1}{n+1}} f(\eta) \\
& \theta(\eta)=\frac{T-T_{\infty}}{T_{f}-T_{\infty}}, \phi(\eta)=\frac{C-C_{\infty}}{C_{w}-C_{\infty}}
\end{aligned}
$$

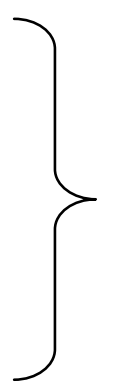

where $\eta$ is the similarity variable and $f(\eta)$ is the dimensionless stream function and $f^{\prime}, \theta$ and $\phi$ are the dimensionless velocity, temperature and concentration respectively and $\psi$ is the Stokes stream function.

The continuity equation is satisfied identically while Eqs.(2)-(4) with the help of Eqs.(5)-(6), become 


$$
\begin{aligned}
& A f^{\prime \prime \prime}+n\left(-f^{\prime \prime}\right)^{n-1} f^{\prime \prime \prime}+\frac{s(2 n-1)+1}{n+1} f f^{\prime \prime}-s\left(f^{\prime}\right)^{2}-M^{2} f^{\prime}=0, \\
& \theta^{\prime \prime}+\frac{s(2 n-1)+1}{n+1} \operatorname{Pr} f \theta^{\prime}+\left(A^{*} f^{\prime}+B^{*} \theta\right)+D u \operatorname{Pr} \phi^{\prime \prime}=0, \\
& \phi^{\prime \prime}+\frac{s(2 n-1)+1}{n+1} S c f \phi^{\prime}+S c \operatorname{Sr} \theta^{\prime \prime}=0,
\end{aligned}
$$

The dimensionless boundary conditions are given by

$$
\begin{aligned}
& f(0)=0, f^{\prime}(0)=1, f^{\prime}(\infty)=0, \\
& \theta^{\prime}(0)=-\gamma[1-\theta(0)], \theta(\eta) \rightarrow 0 \text { as } \eta \rightarrow \infty, \\
& \phi^{\prime}(0)=1, \phi(\infty) \rightarrow 0 \text { as } \eta \rightarrow \infty
\end{aligned}
$$

In equations (7)-(12), differentiation with respect to $\eta$ is represented by primes.Further, for Sisko fluid, $v=\frac{\mu}{\rho}$ is the kinematic viscosity, $\mu$ is the dynamic viscosity. $A$ is the material parameter, $M$ is the magnetic parameter, $\operatorname{Re}_{a}$ and $\mathrm{Re}_{b}$ are the local Reynolds numbers corresponding to Newtonian and power-law fluids, $D_{m}$ is the mass diffusivity, $S r$ is the Soret number, $S c$ is the Schmidt number, $D u$ is the Dufour number, $\operatorname{Pr}$ is the generalized Prandtl number and $\gamma$ is the generalized Biot number appearing in the above equations are defined as follows:

$$
\left.\begin{array}{l}
M^{2}=\frac{\sigma B_{0}^{2}}{\rho U} x, A=\frac{\operatorname{Re}_{b}^{\frac{2}{n+1}}}{\operatorname{Re}_{a}}, \operatorname{Re}_{a}=\frac{\rho x U}{a}, \quad \operatorname{Re}_{b}=\frac{\rho x^{n} U^{2-n}}{b} \quad, \quad \operatorname{Pr}=\frac{x U}{\alpha} \operatorname{Re}_{\left.\right|^{2+1}}^{-2} \\
S r=\frac{D_{m} K_{T}\left(T_{f}-T_{\infty}\right)}{v T_{m}\left(C_{w}-C_{\infty}\right)}, D u=\frac{D_{m} K_{T}\left(C_{w}-C_{\infty}\right)}{v C_{s} C_{p}\left(T_{f}-T_{\infty}\right)}, S c=\frac{v}{D_{m}} \text { and } \gamma=\frac{h_{f}}{k} x \operatorname{Re}_{b}^{\frac{-1}{n+1}}
\end{array}\right\}
$$

The significant quantitities of interest are the skin-friction coefficient $C_{f}$, the local Nusselt number $N u_{x}$ and Local Sherwood number $S h_{x}$ are defined by

$$
C_{f}=\frac{\tau_{w}}{\rho\left(U_{\infty}^{2} / 2\right)}, N u_{x}=\frac{x q_{w}}{k\left(T_{f}-T_{\infty}\right)}, S h_{x}=\frac{x m_{w}}{D_{m}\left(T_{f}-T_{\infty}\right)}
$$

Where $\tau_{w}$ is the surface shear stress, $q_{w}$ is the surface heat flux and $m_{w}$ is the rate of mass transfer and are defined by,

$$
\tau_{w}=\mu\left(\frac{\partial u}{\partial y}\right)_{y=0}, q_{w}=-k\left(\frac{\partial T}{\partial y}\right)_{y=0}, m_{w}=-D_{m}\left(\frac{\partial C}{\partial y}\right)_{y=0}
$$

using the non-dimensional variables, we obtain, 


$$
\begin{aligned}
& \frac{1}{2} \mathrm{Re}_{b}^{\frac{1}{n+1}} C_{f}=A f^{\prime \prime}(0)-\left[-f^{\prime \prime}(0)\right]^{n}, \\
& \operatorname{Re}_{b}^{\frac{-1}{n+1}} N u_{x}=-\theta^{\prime}(0), \\
& S h_{x} \operatorname{Re}_{b}^{\frac{-1}{n+1}}=-\phi^{\prime}(0)
\end{aligned}
$$

\section{RESULTS AND DISCUSSIONS}

The set of transformed ordinary differential Eqns.7-9 with the corresponding boundary conditions 10-12 are solved numerically by using MATLAB bvp4c package. The obtained results on velocity, temperature and concentration fields are presented and analyzed through graphs. Also, the numerical results of Skin friction coefficient, local Nusselt number and local Sherwood number are presented in Tables 1-3. For numerical results we considered $s=0.5, \operatorname{Pr}=7, A^{*}=B^{*}=0.5, n=3, A=1, \gamma=1$,

$S r=D u=S c=0.1$. These values are kept as common throughout our analysis except the varied values are shown in respective figures and tables.

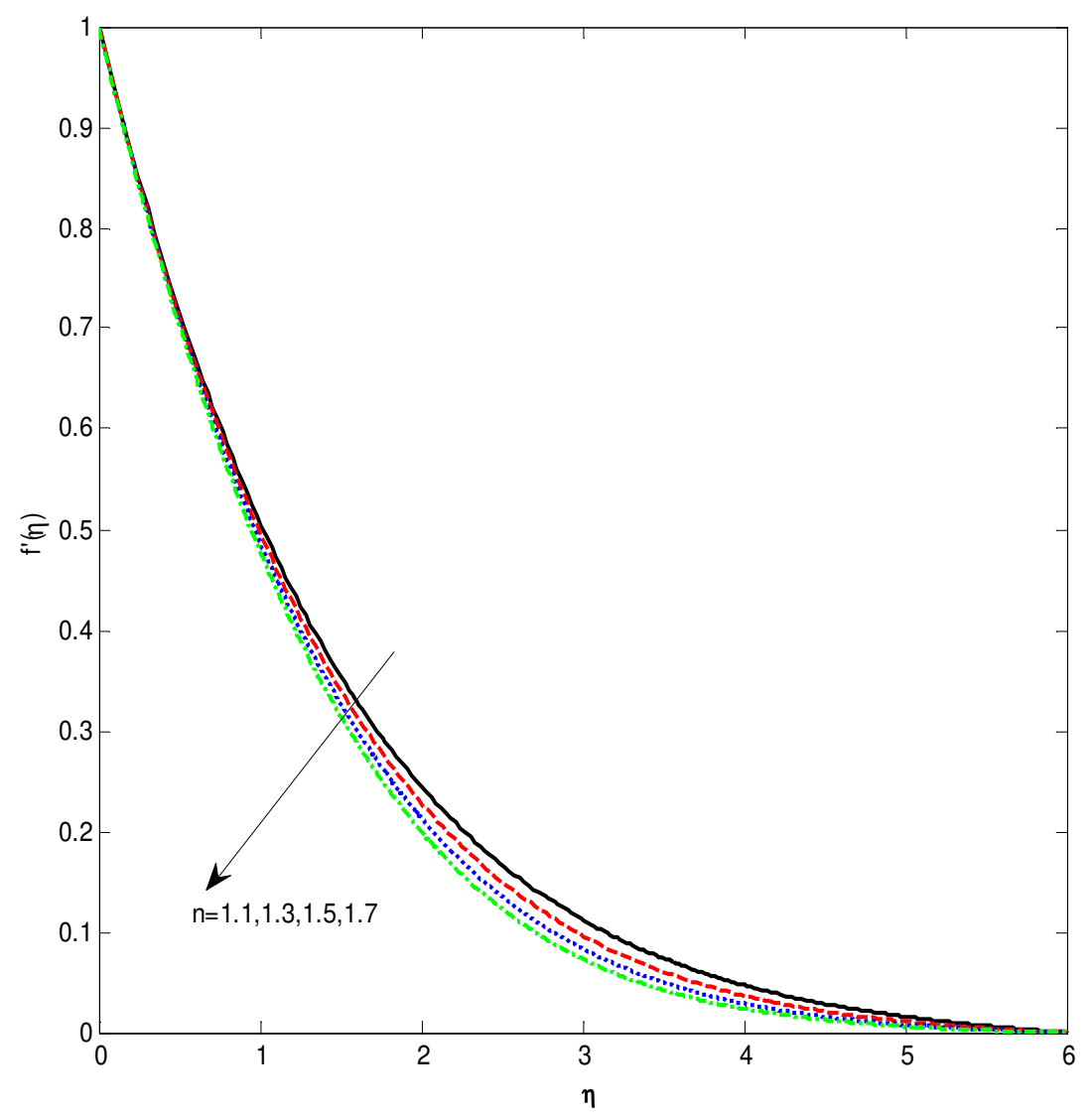

Figure 2: Effect of power law index $n$ on $f^{\prime}(\eta)$ 


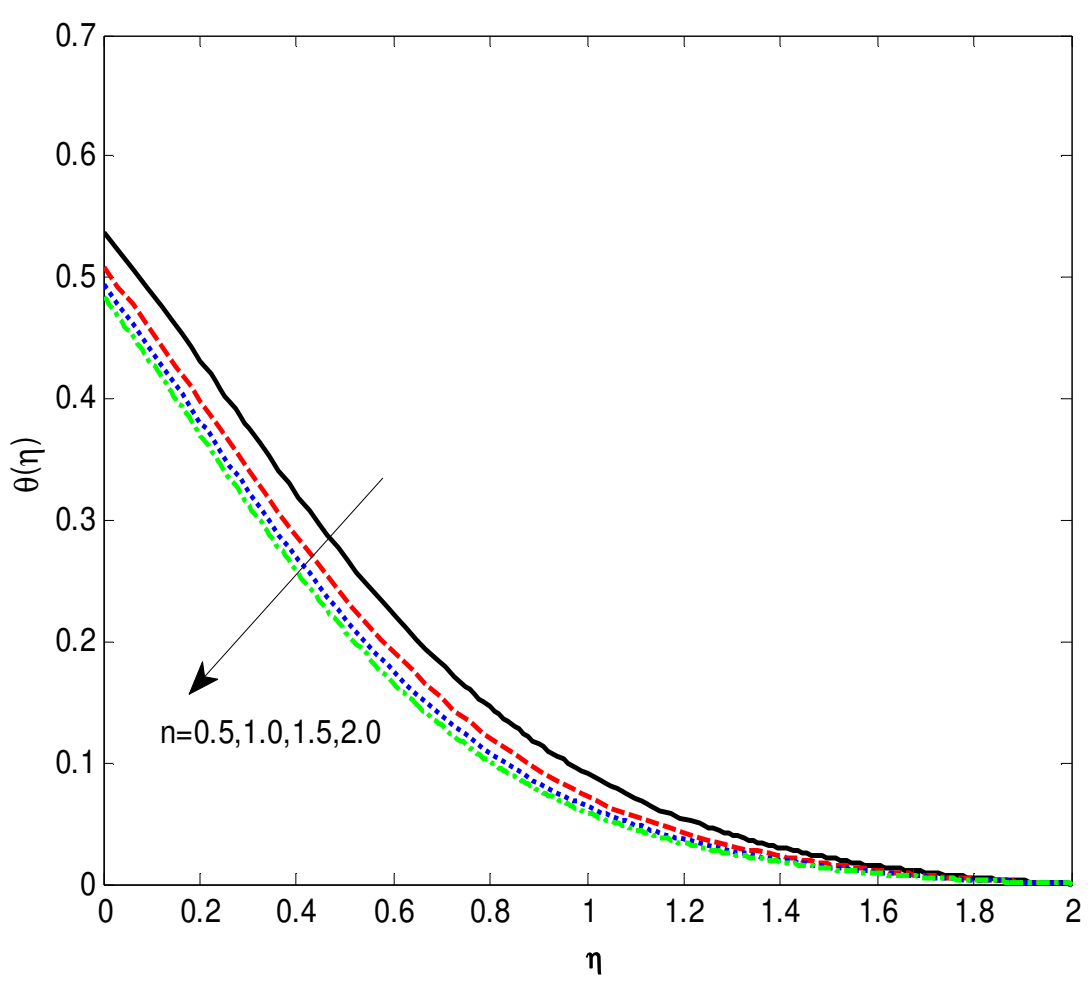

Figure 3: Effect of power law index $n$ on $\theta(\eta)$

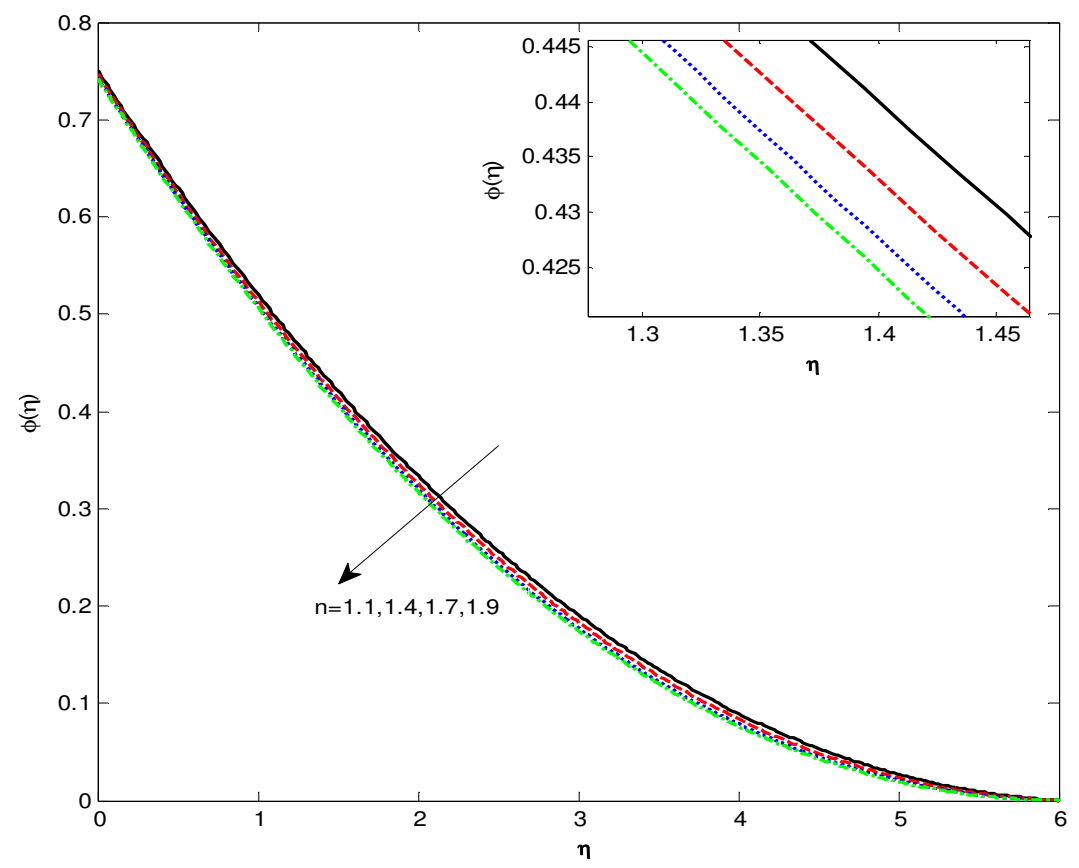

Figure 4: Effect of power law index $n$ on $\phi(\eta)$ 


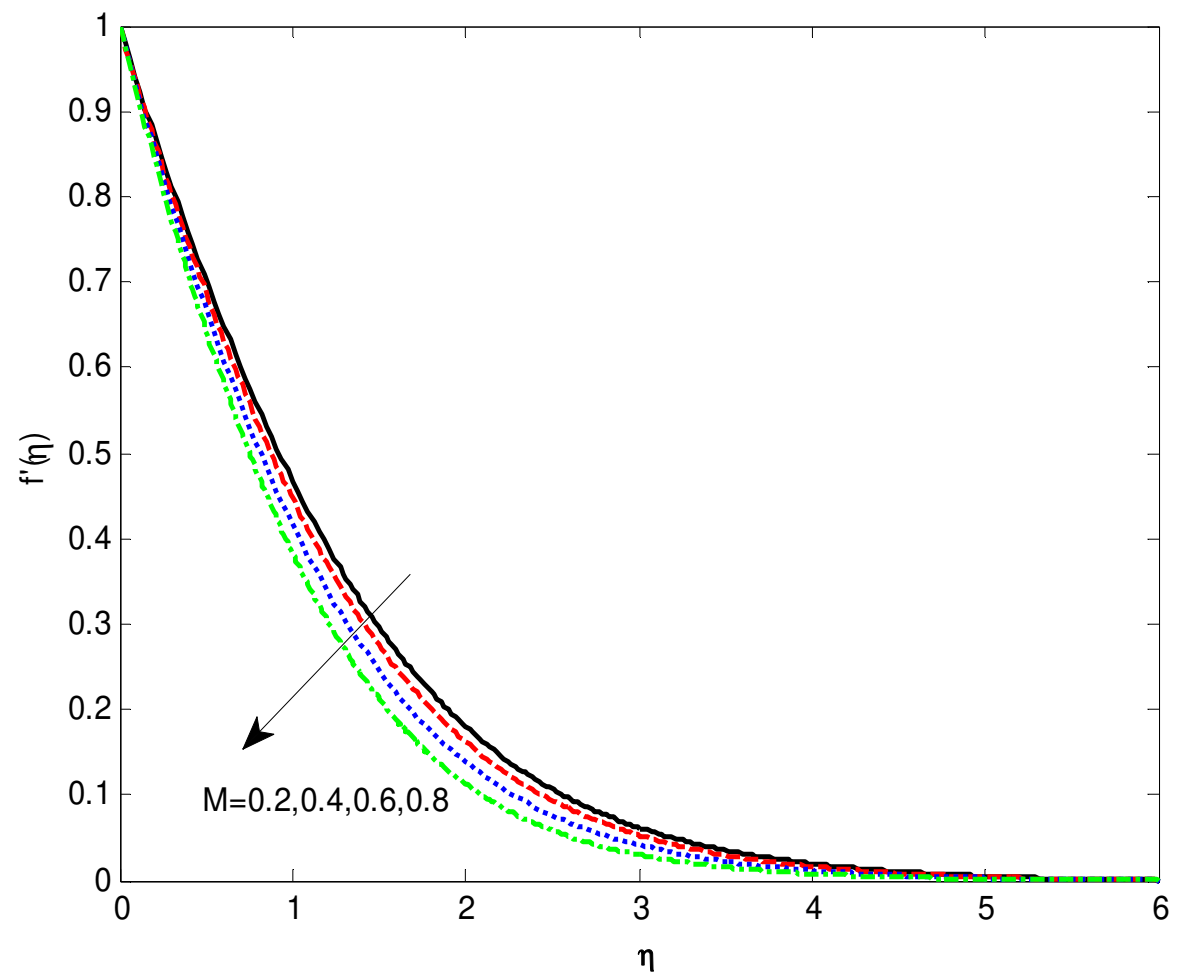

Figure 5: Effect of Magnetic field parameter $M$ on $f^{\prime}(\eta)$

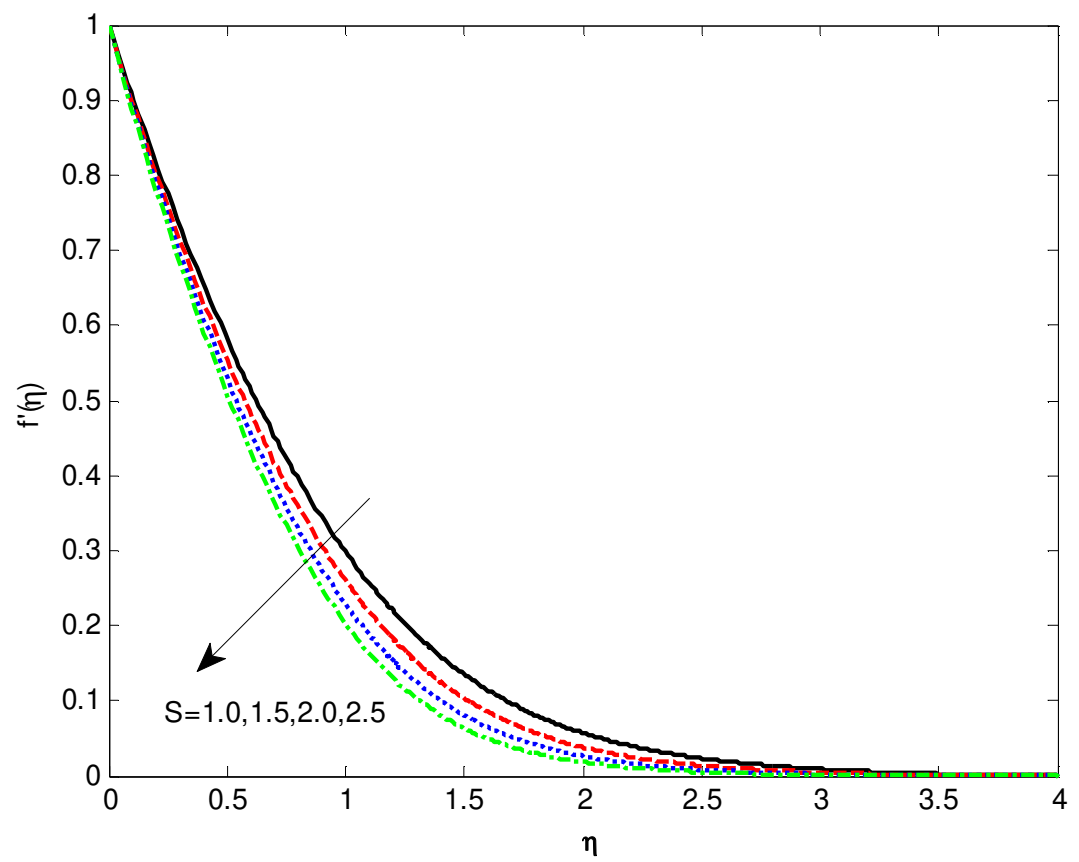

Figure 6: Effect of stretching parameter $s$ on $f^{\prime}(\eta)$ 


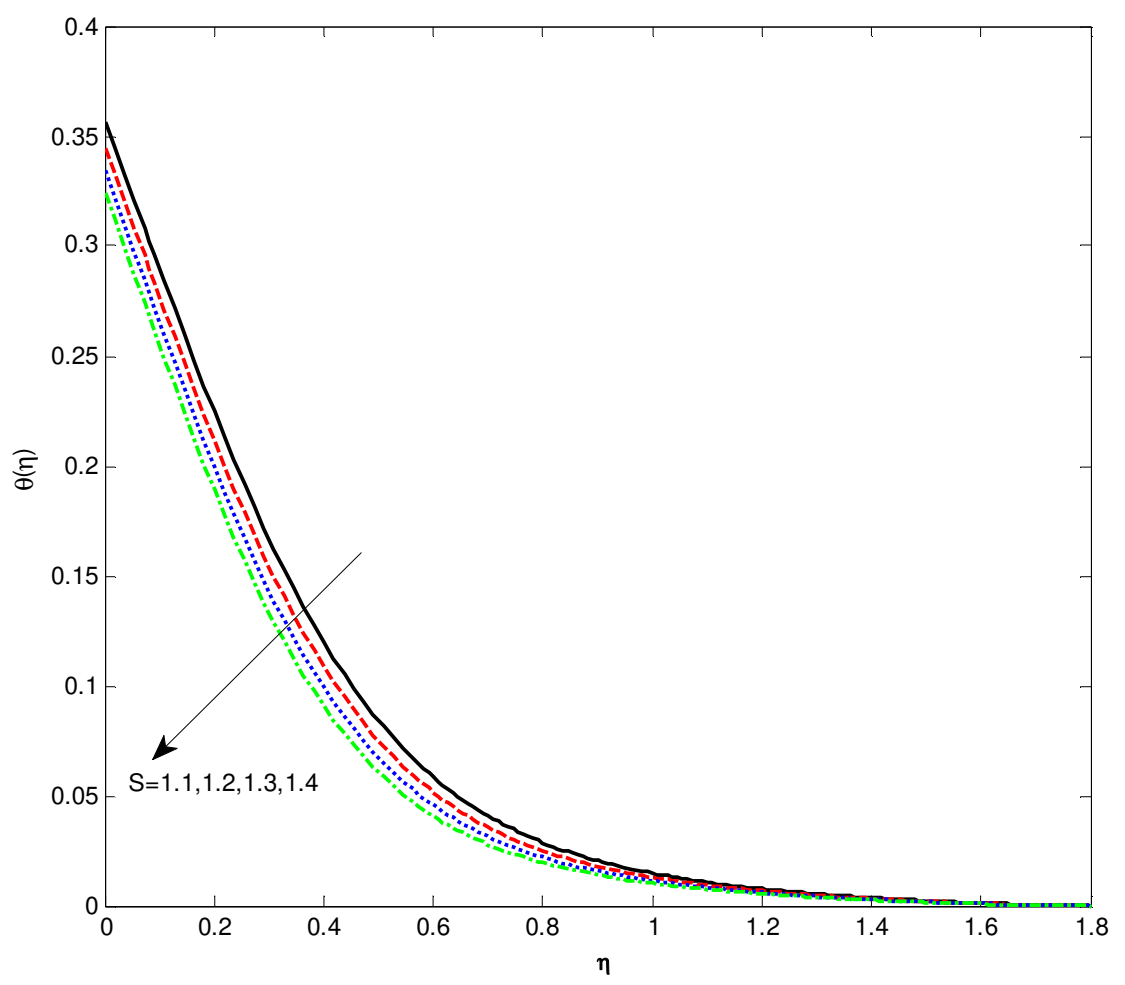

Figure 7: Effect of stretching parameter $s$ on $\theta(\eta)$

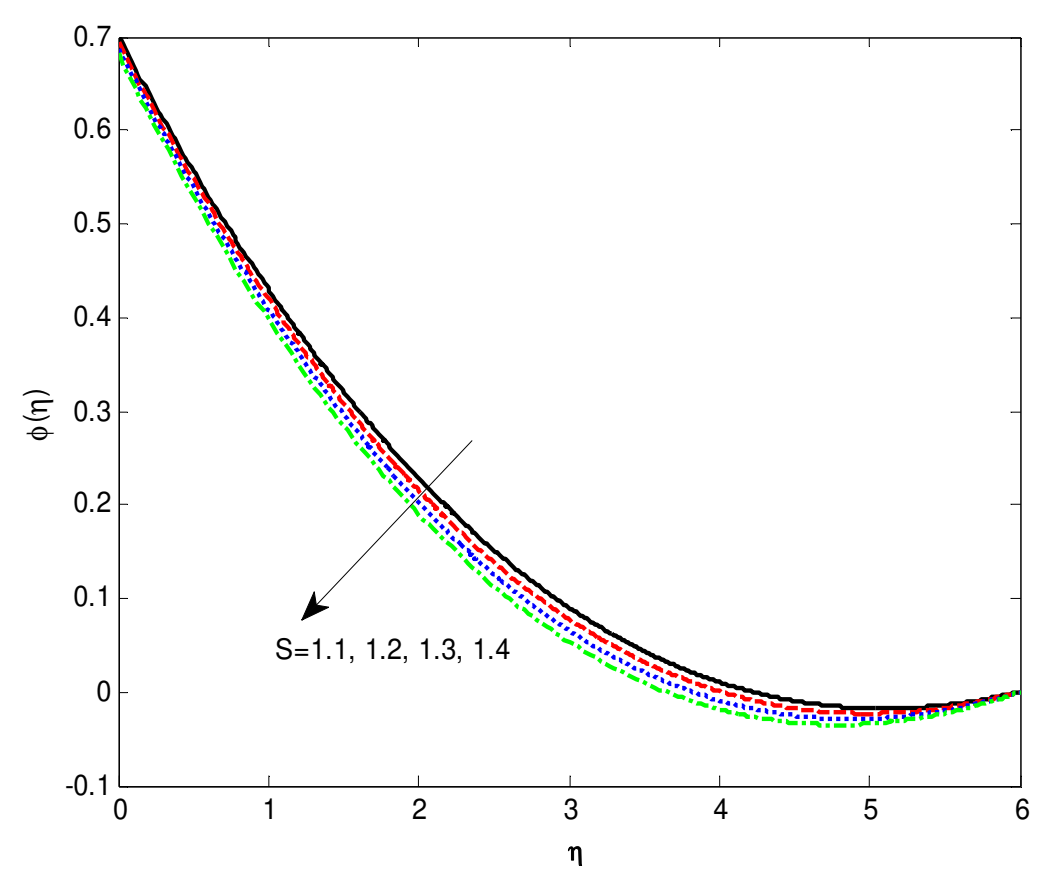

Figure 8: Effect of stretching parameter $s$ on $\phi(\eta)$ for fixed $\mathbf{A}=0.5 ; \gamma=1.5$ 


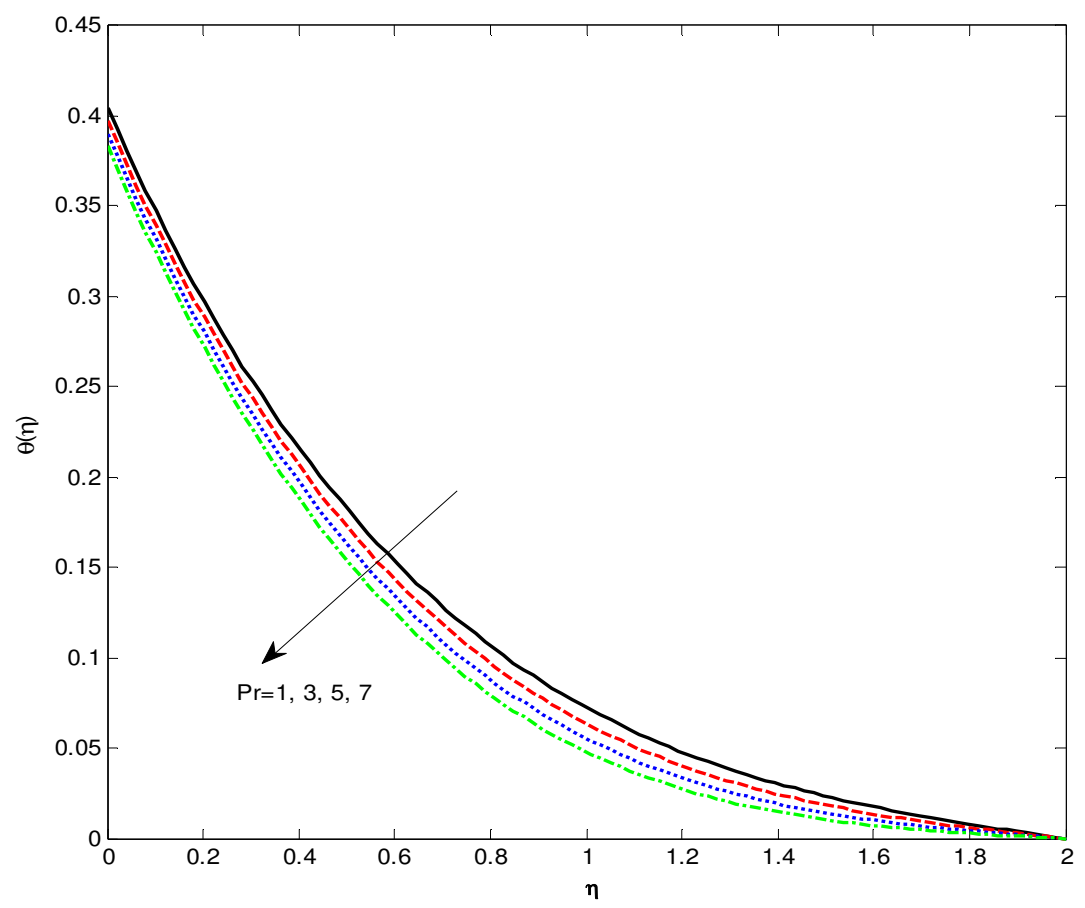

Figure 9: Effect of Prandtl number $\operatorname{Pr}$ on $\theta(\eta)$ for fixed $\mathrm{A}^{*}=\mathrm{B}^{*}=-0.5 ; \mathrm{M}=0.5 ; \mathrm{A}=0.5$

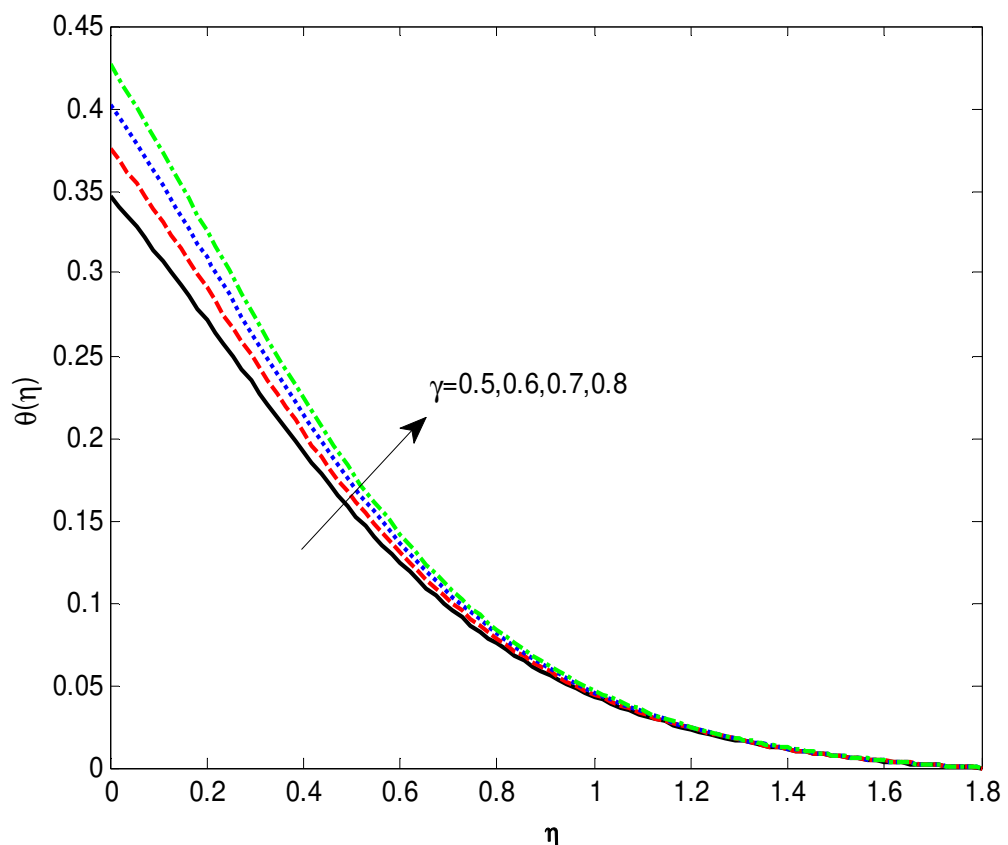

Figure 10: Effect of Biot number $\gamma$ on $\theta(\eta)$ for fixed $\operatorname{Pr}=2 ; \mathrm{M}=1$; Du=0.01; Sr=0.2; Sc=0.1 


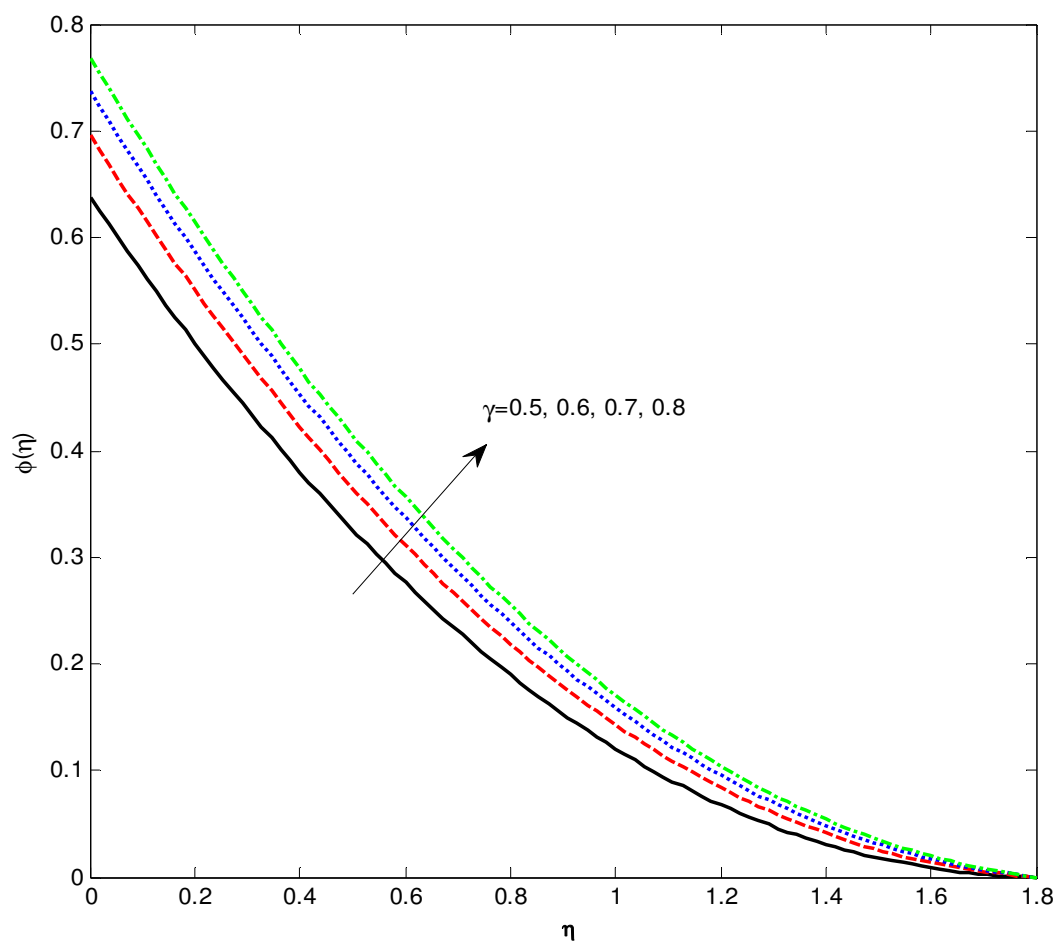

Figure 11: Effect of Biot number $\gamma$ on $\phi(\eta)$ for fixed $\mathbf{A}=\mathbf{0 . 5}$; $\mathbf{S c}=\mathbf{0 . 5}$;

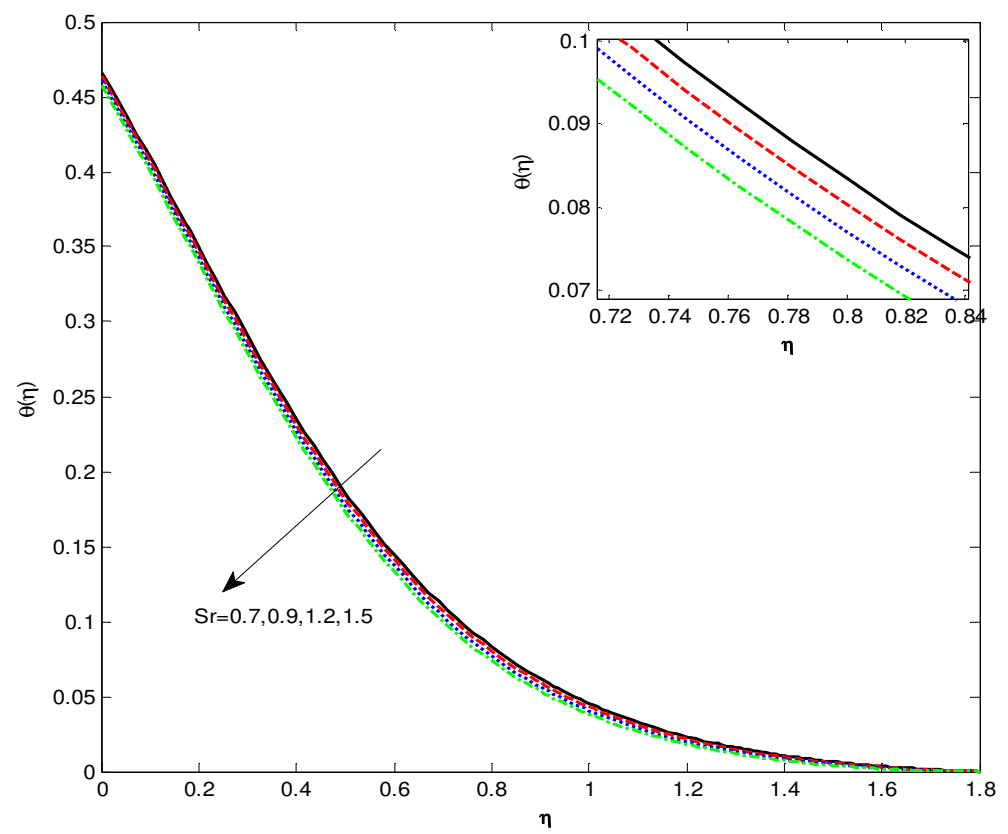

Figure 12: Effect of soret number $\operatorname{Sr}$ on $\theta(\eta)$ 


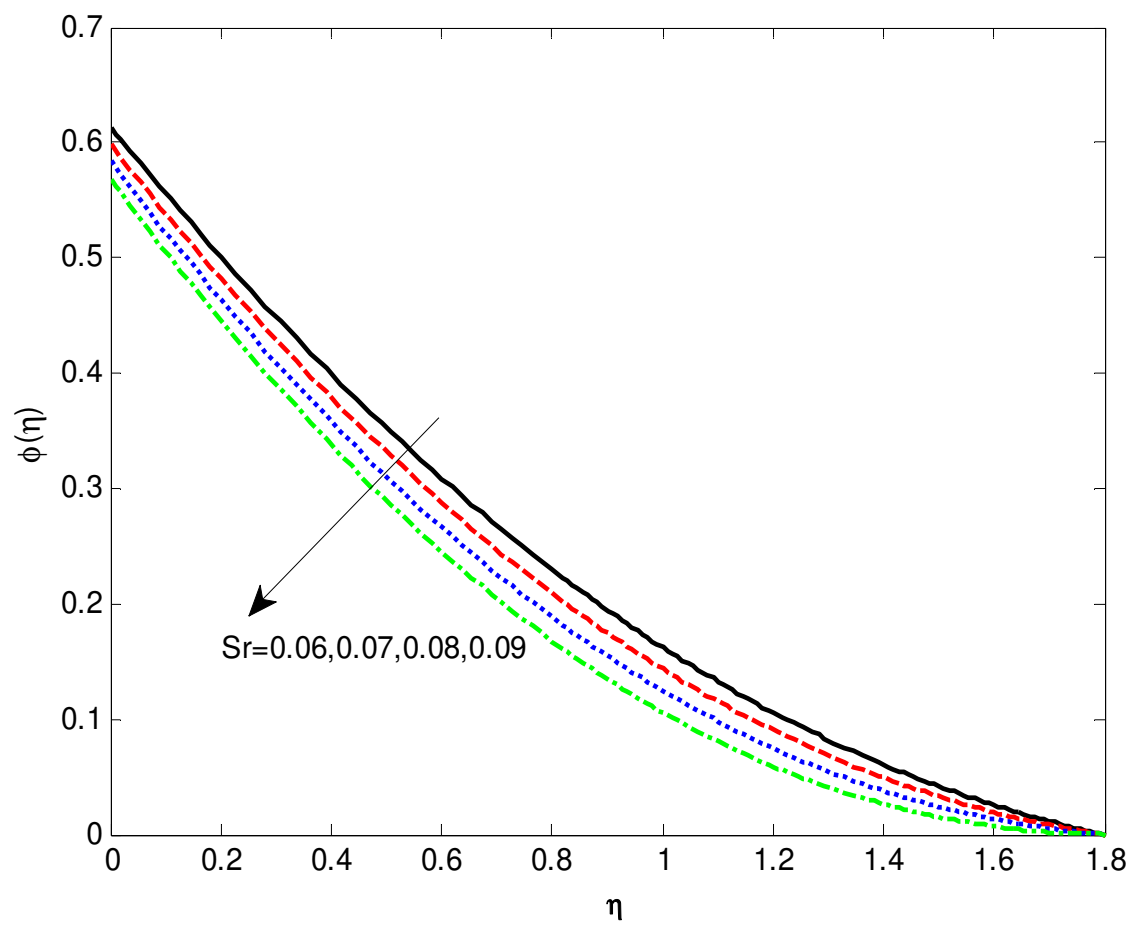

Figure 13: Effect of soret number Sr on $\phi(\eta)$ for fixed $\gamma=1.5$; Sc=0.5

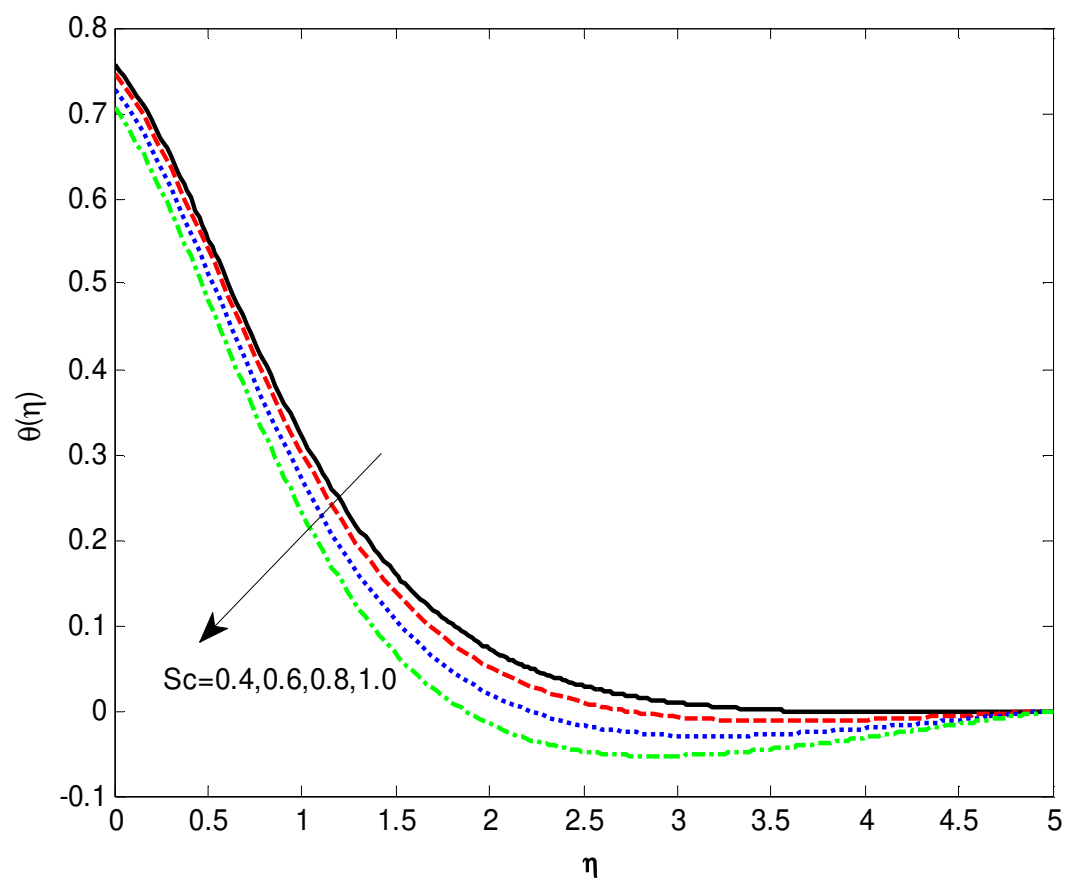

Figure 14: Effect of schmidth number Sc on $\theta(\eta)$ for fixed $\operatorname{Pr}=3$ 


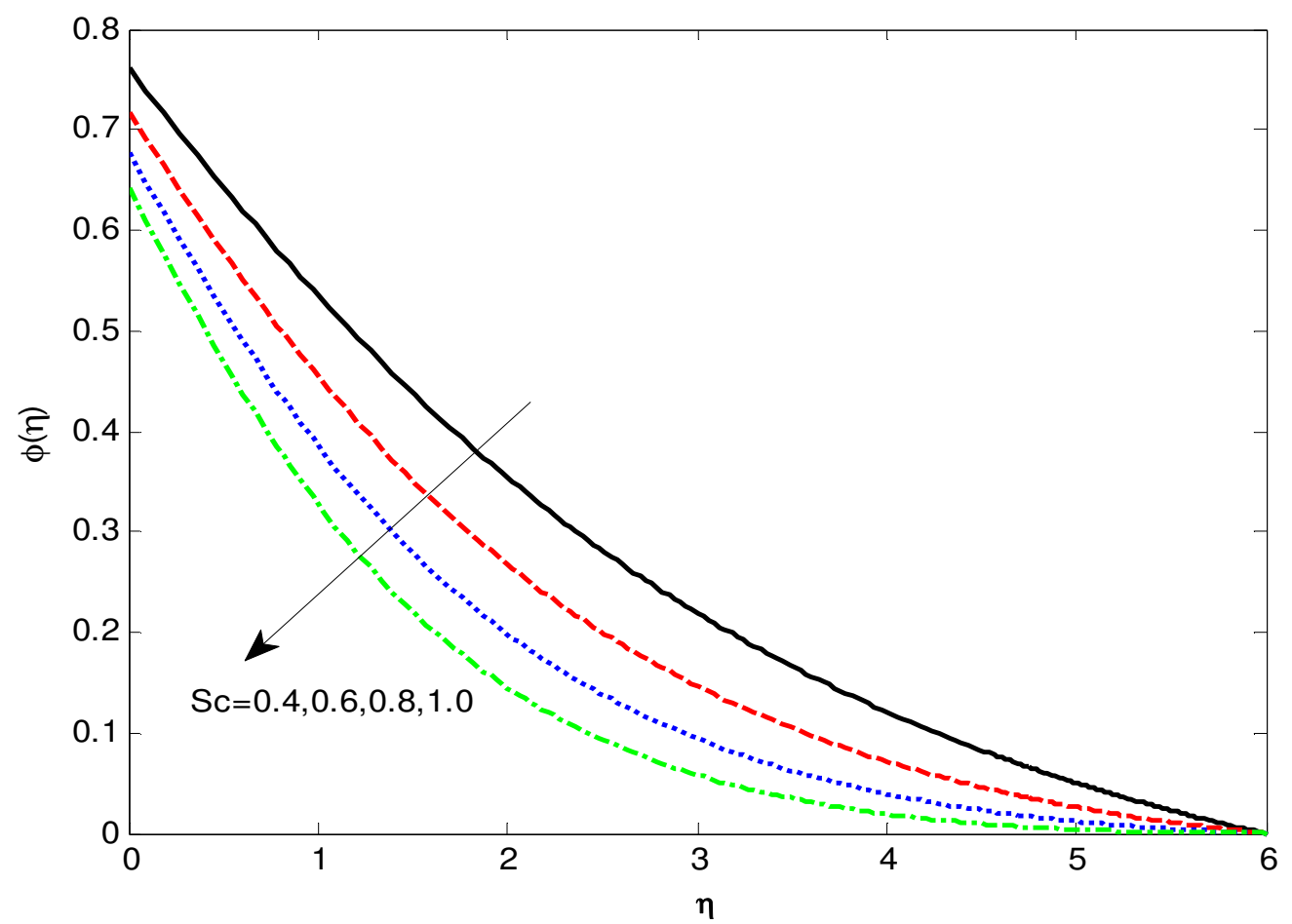

Figure 15: Effect of Schmidth number Scon $\phi(\eta)$ for fixed $\operatorname{Pr}=0.7 ; \operatorname{Sr}=0.01$

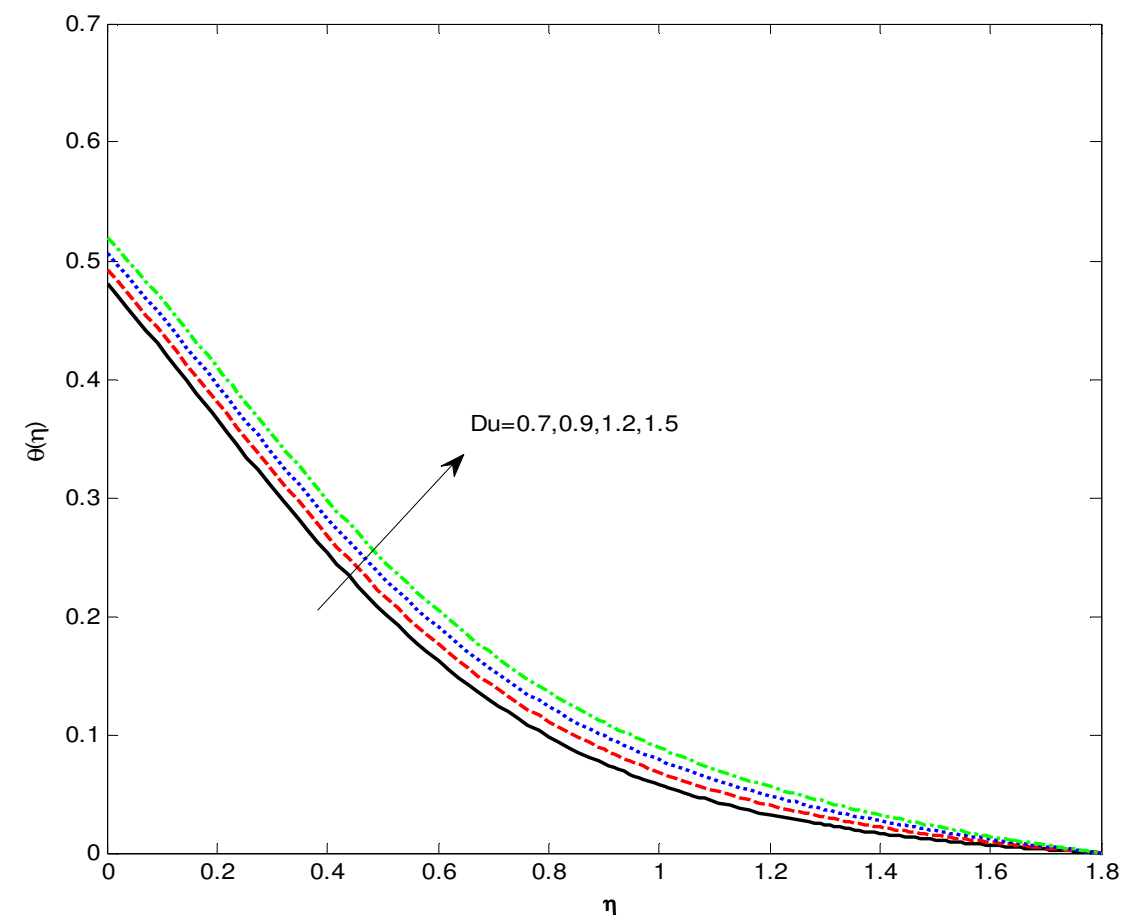

Figure 16: Effect of Dufour number Du on $\theta(\eta)$ 


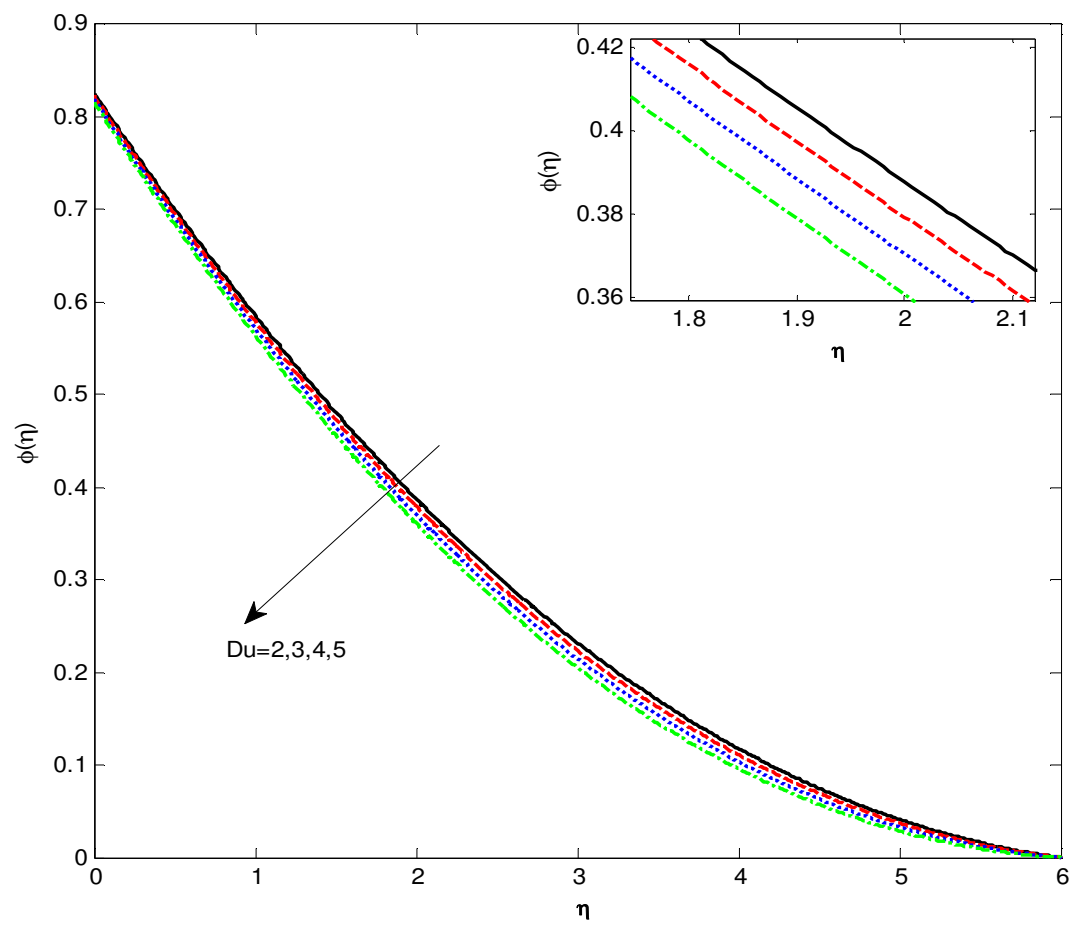

Figure 17: Effect of Dufour number Du on $\phi(\eta)$ for fixed $\gamma=1.5 ; \mathrm{Sr}=\mathbf{0 . 0 6} ; \mathrm{Sc}=\mathbf{0 . 0 8}$

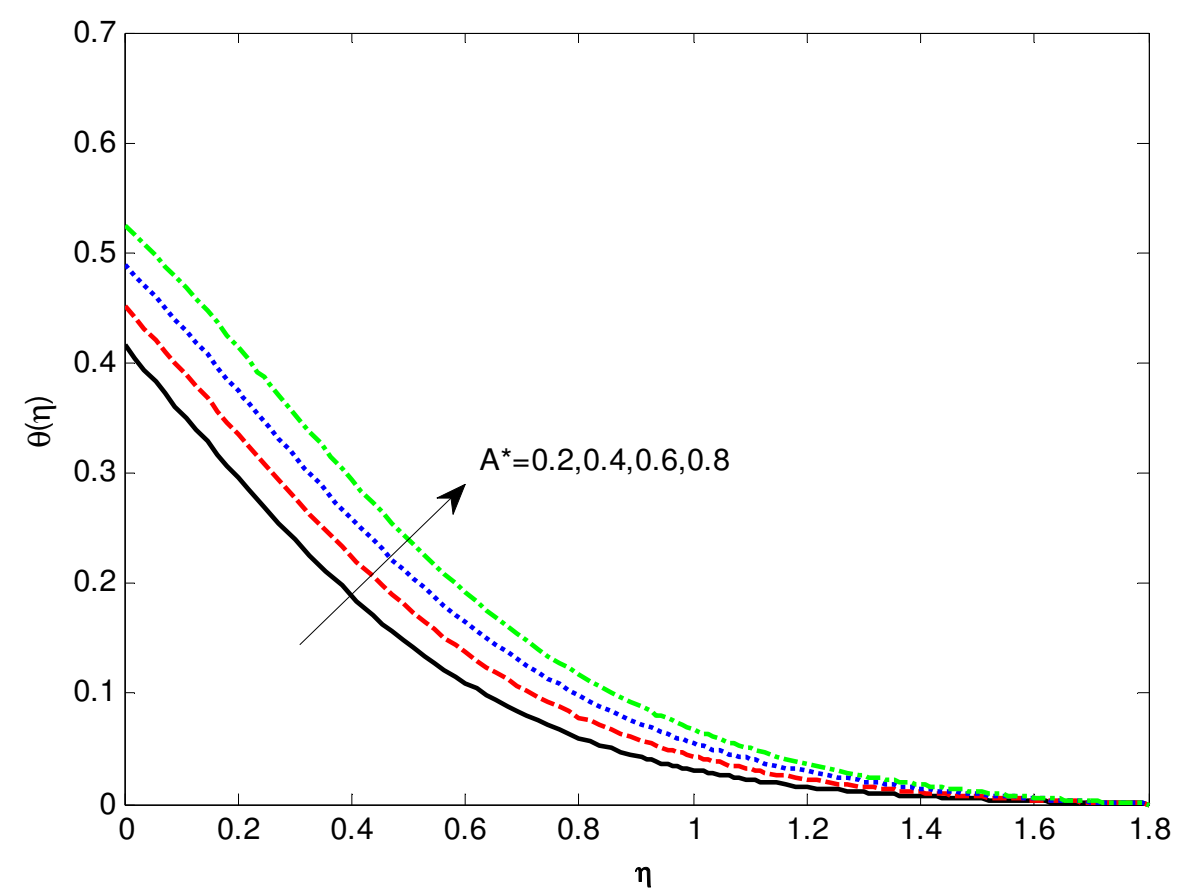

Figure 18: Effect of space dependent heat source/sink parameter $\mathbf{A}^{*}$ on $\theta(\eta)$

for fixed $\mathrm{M}=1.5 ; \gamma=1.5 ; \mathrm{Sc}=0.2$ 


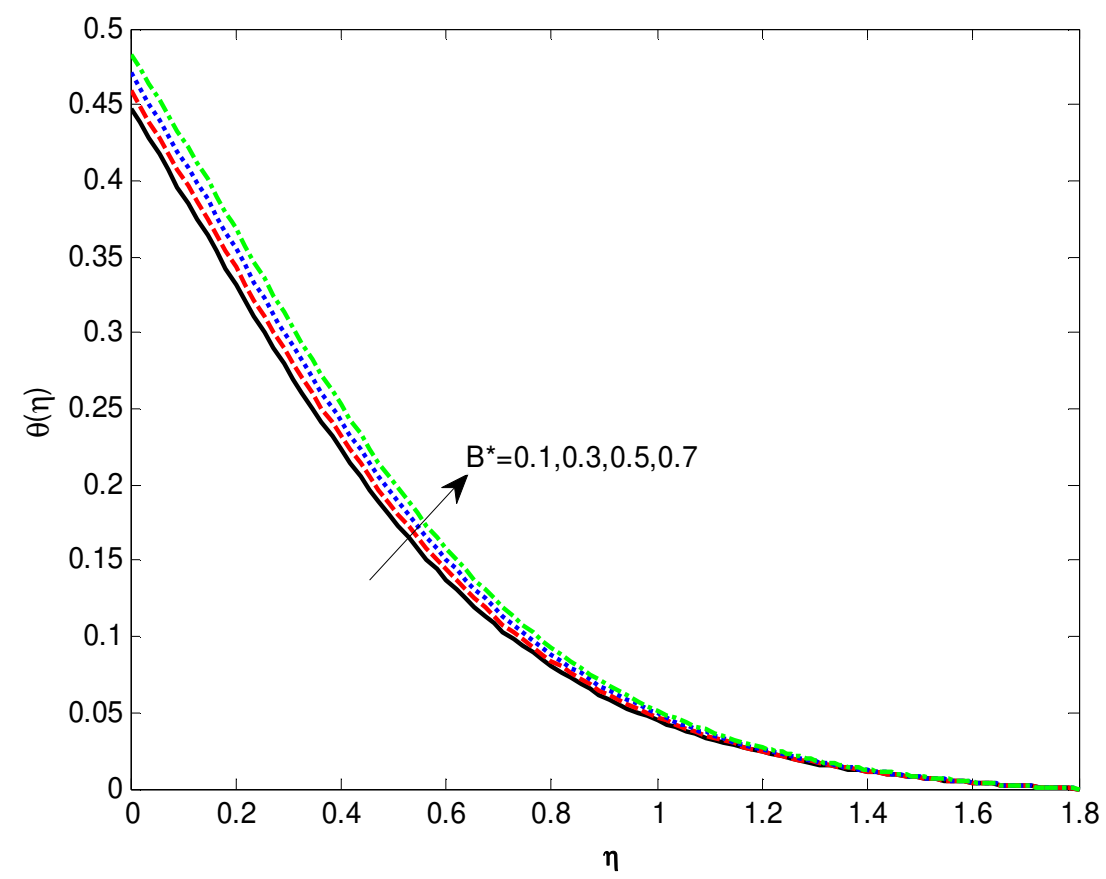

Figure 19: Effect of temperature dependent heat source/sink parameter $\mathbf{B} *$ on $\theta(\eta)$

for fixed $\mathrm{M}=1.5 ; \gamma=1.5 ; \mathrm{Sc}=0.2$

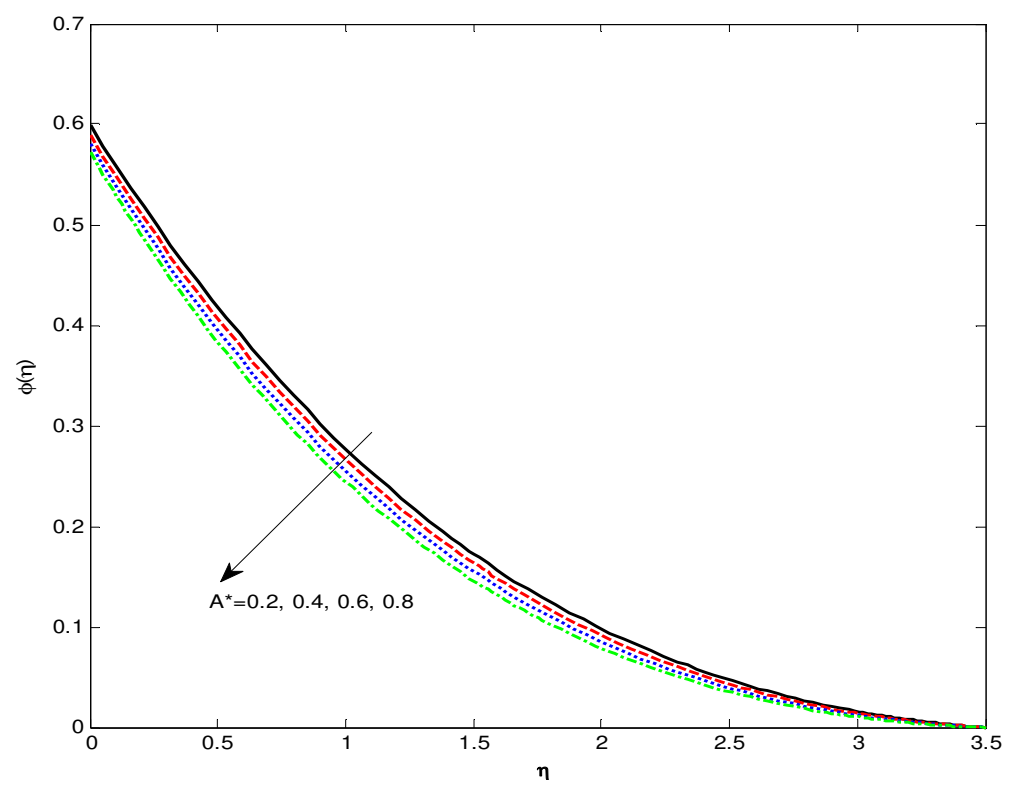

Figure 20: Effect of space dependent heat source/sink parameter $A^{*}$ on $\phi(\eta)$

for fixed $\mathrm{B}^{*}=0.5 ; \gamma=1.5 ; \mathrm{Sc}=0.35$ 


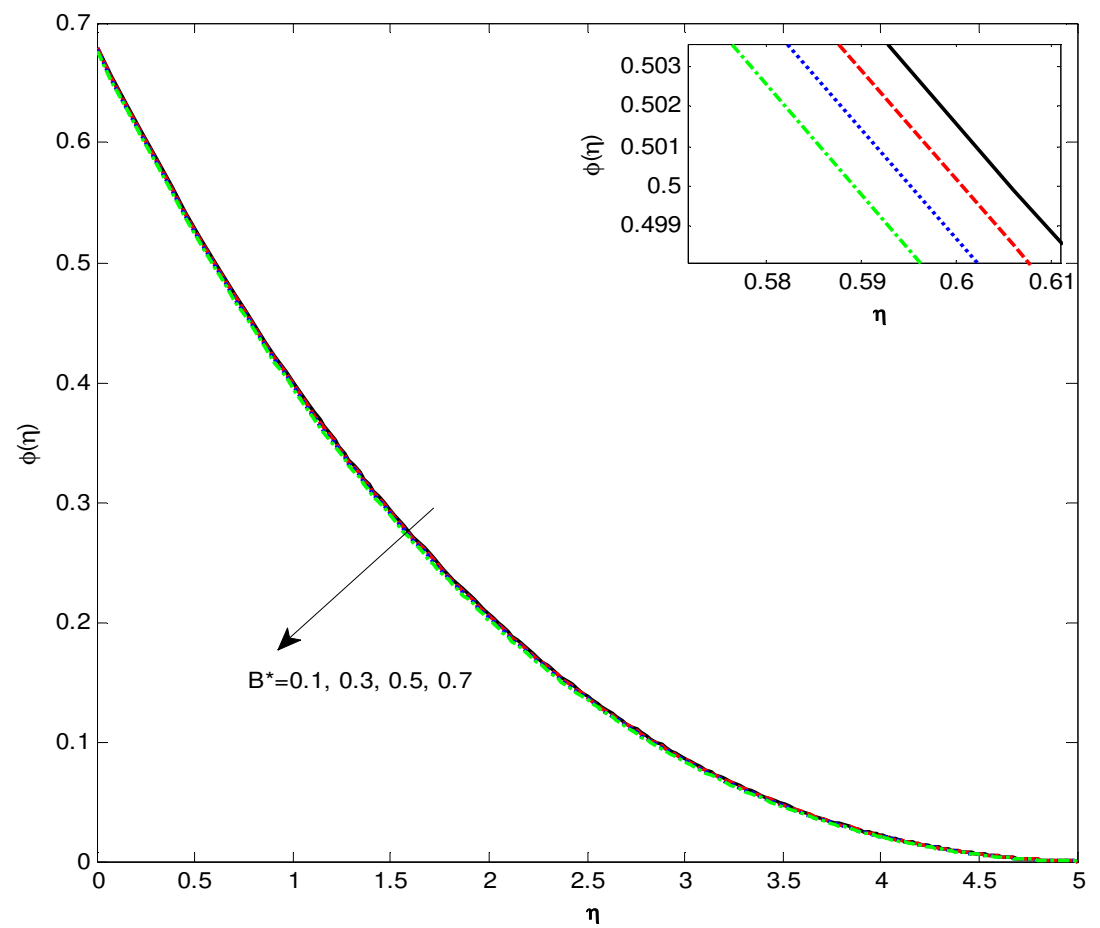

Figure 21: Effect of temperature dependent heat source/sink parameter $B *$ on $\phi(\eta)$

for fixed $\mathrm{M}=1.5 ; \gamma=1.5 ; \mathrm{Sc}=0.2$

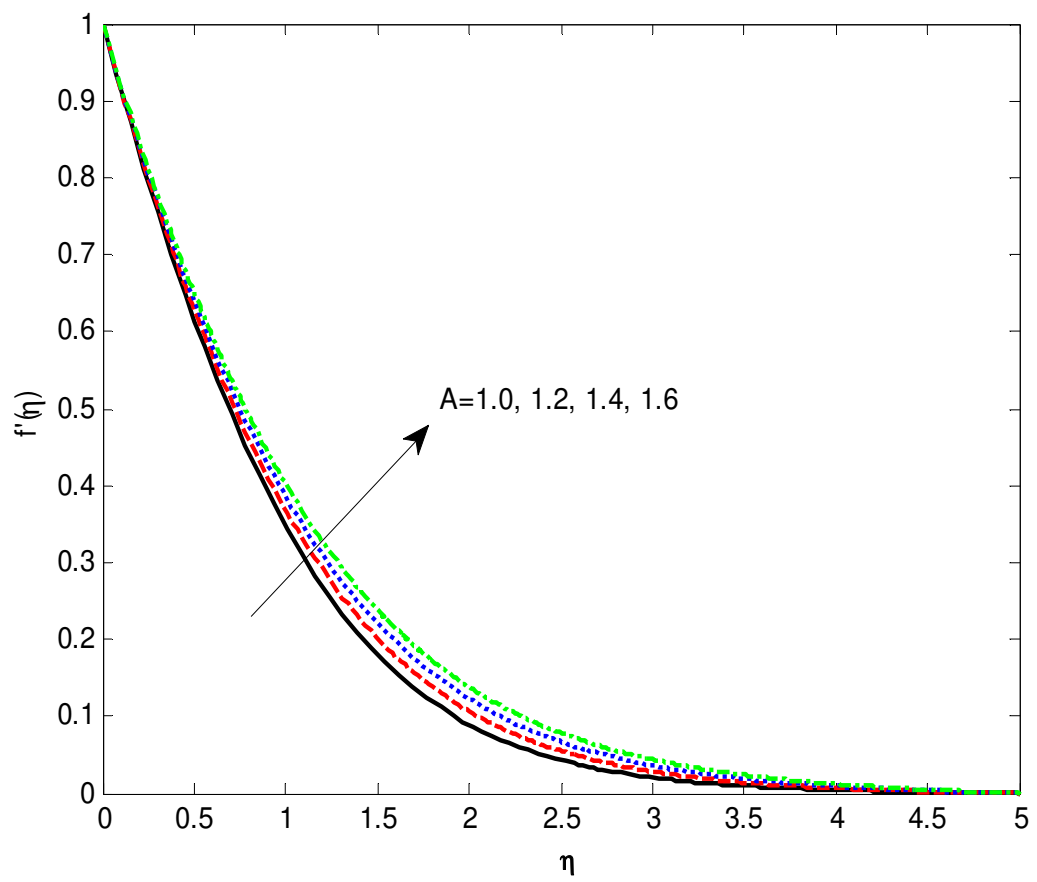

Figure 22: Effect of material parameter $A$ on $f^{\prime}(\eta)$

Fig.2 illustrates the influence of the power-law index $n$ on velocity field $\left(f^{\prime}(\eta)\right)$. We noticed a decrement 
behavior in fluid velocity with an increase in power-law index. Fig.3 portrays the effect of the power law index on fluid temperature, from this figure; it is obvious that the temperature field decreases with an increase in power-law index. Also, the thermal boundary layer thickness decreases. The effect of power law index number $n$ on concentration profile is presented in Fig.4. It is seen that increasing value of $n$ lessens the concentration distribution. The effect of magnetic field parameter $(M)$ on velocity is shown in Fig.5, it is seen that the fluid velocity decreases with an increase in M.This is because of the presence of Lorentz force, which has a tendency to reduce the motion of fluid, hence the velocity profiles decreases with the effect of magnetic field parameter.Figs.6-8 demonstrates the effect of stretching parameter $(s)$ on the velocity, temperature and concentration distributions respectively. From these figures it is noticed that velocity, temperature and concentration decrease with the increasing value of stretching parameter. The effect of the Prandtl number (Pr) on the dimensionless temperature is illustrated in Fig.9 it can be seen that the dimensionless temperature reduces for higher values of Pr. Physically, Prandtl number is the ratio of momentum and thermal diffusivities. Thus, higher Prandtl number corresponds to the lower thermal diffusivity, which is responsible in reduction of the temperature profile.The graph of $\theta(\eta)$ vs. $\eta$ for different values of the generalized Biot number $(\gamma)$ is presented through Fig.10. An observation reveals that the temperature field $\theta(\eta)$ increases rapidly near the boundary for a larger Biot number. Physically, an increase in $\gamma$ declines the resistance of hot fluid convection. As a result the surface temperature along with the thermal boundary layer thickness increases.It can easily be seen from Fig.11 that $\phi(\eta)$ increases with the increasing value of $\gamma$. From Fig.12 it is clear that the temperature distribution across the thermal boundary layer thickness reduces with increase of $\mathrm{Sr}$. The reason behind this phenomenon is that, higher values of $S r$ reduces the thermal diffusivity. From Fig.13 we notice that concentration field decreases for rising values of $S r$. Generally higher values of $S r$ enhances the fluid concentration. But due to the domination of stretching parameter, we observe the result of that kind.It is observed from Fig.14 that $\theta(\eta)$ decreases for higher value of $S c$. Also from Fig.15 it is seen that the fluid concentration decreases with the increasing value of Schmidt number $(S c)$. This is due to the fact that the increasing value of $S c$ causes a reduction in molecular diffusivity. Hence, the concentration of the species is higher for large values of $S c$ and lower for small values of $S c$.

It is noticed from Figs.16 and 17 that as Dufour number increases, temperature decreases and concentration increases. The Dufour effect is the energy flux due to a mass concentration gradient occurring as a coupled effect of irreversible processes. It is the reciprocal phenomenon to the Soret effect. The concentration gradient results in a temperature change. So improving the Dufour number, downturns the temperature and rises the concentration.Figs.18-21 illustrate the effect of non-uniform heat source/sink parameters $\left(A^{*}, B^{*}\right)$ on the temperature and concentration profiles of the flow. It is evident from the figures that an increase in the values of $A^{*}$ or $B^{*}$ enhances the temperature profiles but reduces the concentration profiles. This may happen due to the fact that the positive values of $A^{*}, B^{*}$ acts like heat generators. Generating the heat means release of heat energy to the flow. This help to enhance the thermal boundary layer thickness and suppress the concentration boundary layer thickness. It is clear that the effect of non-uniform heat source/sink is more on fluid temperature than that of concentration.Fig.22 describes the influence of material parameter $A$ on the velocity profile. From these figures it is observed that an increase in the material parameter $A$ causes an increase in the velocity and boundary layer thickness. 
The numerical values of the Skin friction coefficient $\left(\frac{1}{2} \operatorname{Re}_{b}^{\frac{1}{n+1}} C_{f x}\right)$, Local Nusselt number $\left(\operatorname{Re}_{b}^{\frac{-1}{n+1}} N u_{x}\right)$ and Sherwood number $\left(\operatorname{Re}_{b}^{\frac{-1}{n+1}} S h_{x}\right)$ for different flow parameters with variation of

Power law index parameter $(n)$ is presented in Tables 1-3. The effect of magnetic field parameter $(M)$, power law index $(n)$, stretching rate parameter $(s)$ and material parameter $(A)$ on Skin friction coefficient $\left(C_{f}\right)$ is shown in Table 1 , through numerical values. It is obvious that Skin friction $\left(C_{f}\right)$ increases with increasing values of power law index. But we notice a decrease in Skin friction for rising value of magnetic field parameter $(M)$, stretching rate parameter $(s)$ and material parameter $(A)$.

Further the effects of various parameters such as Dufour number $(D u)$, Soret number $(S r)$, Prandtl number $(\operatorname{Pr})$ and non uniform heat sourse/sink parameters on heat transfer rate is shown in Table 2. It is noticed that Dufour effect causes lesser heat transfer rate but the result is quite opposite with Soret effect. Increase in stretching rate of the surface also helps to enhance the heat rate of the surface also helps to enhance the heat transfer parameter. It is clear that the heat source caused by $A^{*}>0, B^{*}>0$ reduces the nusselt number significantly. Our results well agree with the fact that increases in Prandtl number $(\operatorname{Pr}) /$ power law index $(n)$ causes higher rate of heat transfer.The influence of various parameters (which are already discussed in Table 2 for nusselt number) on mass transfer rate can be visualized in Table 3. It is apparent that Dufour and Soret effects exhibit opposite results on mass transfer rate. An increase in Schmidt number (or) stretching rate parameter causes an enhancement in mass transfer performance. Also, the heat energy caused by $A^{*}>0, B^{*}>0$ reduces the sherwood number. We note that the effect of Schmidt number and Dufour number on mass transfer rate is notable.

\section{CONCLUSIONS}

- An increase in magnetic field parameter $(M)$, stretching rate parameter $(s)$ and power law index $(n)$ slows down the fluid velocity.

- Heat source due to $A^{*}>0, B^{*}>0$ reduce the heat transfer rate but upgrades the mass transfer rate effectively.

- Dufour and Soret effects play a vital role in deciding the heat and mass transfer rates.

- Increase in material parameter $(A)$, stretching rate parameter $(s)$ causes a reduction in friction factor.

- Increase in Biot number $(\gamma)$ boosts the fluid temperature and concentration.

- Nusselt and Sherwood numbers are increasing function of power law index $(n)$. 
Table: 1 Numerical values of the skin friction coefficient $\frac{1}{2} \operatorname{Re}_{b}^{\frac{1}{n+1}} C_{f x}$ for different values of physical parameters

\begin{tabular}{|l|l|l|l|l|}
\hline $\mathbf{A}$ & $\mathbf{M}$ & $\mathbf{S}$ & $\mathbf{n}$ & $\frac{1}{2} \operatorname{Re}_{b}^{1 / n+1} C_{f x}$ \\
\hline $\mathbf{1 . 0}$ & & & & -1.558918 \\
\hline $\mathbf{1 . 2}$ & & & & -1.642127 \\
\hline $\mathbf{1 . 4}$ & & & & -1.723398 \\
\hline $\mathbf{1 . 6}$ & & & & -1.802761 \\
\hline & $\mathbf{0 . 2}$ & & & -0.941336 \\
\hline & $\mathbf{0 . 4}$ & & & -1.028660 \\
\hline & $\mathbf{0 . 6}$ & & & -1.166288 \\
\hline & $\mathbf{0 . 8}$ & & & -1.345615 \\
\hline & & $\mathbf{1 . 0}$ & & -1.875110 \\
\hline & & $\mathbf{1 . 5}$ & & -2.169058 \\
\hline & & $\mathbf{2 . 0}$ & & -2.446653 \\
\hline & & & $\mathbf{1 . 1}$ & -1.760464 \\
\hline & & & $\mathbf{1 . 3}$ & -1.722475 \\
\hline & & & $\mathbf{1 . 5}$ & -1.691007 \\
\hline & & & $\mathbf{1 . 7}$ & -1.664476 \\
\hline
\end{tabular}

Table 2: Numerical values of the local Nusselt number $\operatorname{Re}_{b}^{\frac{-1}{n+1}} N u_{x}$ for different values of physical parameters

\begin{tabular}{|c|c|c|c|c|c|c|c|}
\hline $\mathbf{D u}$ & $\mathrm{Sr}$ & $\bar{n}$ & $\mathbf{S}$ & $\mathbf{P r}$ & $\mathbf{A}^{*}$ & $\mathrm{~B}^{*}$ & $\mathrm{Re}_{b}{ }^{-1 / n+1} N u_{x}$ \\
\hline 0.7 & & & & & & & 0.490105 \\
\hline 0.9 & & & & & & & 0.482291 \\
\hline 1.2 & & & & & & & 0.470704 \\
\hline 1.5 & & & & & & & 0.459298 \\
\hline & 0.7 & & & & & & 0.528422 \\
\hline & 0.9 & & & & & & 0.533561 \\
\hline & 1.2 & & & & & & 0.541576 \\
\hline & 1.5 & & & & & & 0.549980 \\
\hline & & 0.5 & & & & & 0.429552 \\
\hline & & 1.0 & & & & & 0.467226 \\
\hline & & 1.5 & & & & & 0.488056 \\
\hline & & & 1.1 & & & & 0.638548 \\
\hline & & & 1.2 & & & & 0.651129 \\
\hline & & & 1.3 & & & & 0.662466 \\
\hline & & & 1.4 & & & & 0.672757 \\
\hline & & & & 3 & & & 0.246173 \\
\hline & & & & 5 & & & 0.427550 \\
\hline & & & & 7 & & & 0.513907 \\
\hline & & & & & 0.2 & & 0.575359 \\
\hline & & & & & 0.4 & & 0.534391 \\
\hline & & & & & 0.6 & & 0.493423 \\
\hline & & & & & 0.8 & & 0.452455 \\
\hline & & & & & & 0.1 & 0.539790 \\
\hline & & & & & & 0.3 & 0.527374 \\
\hline & & & & & & 0.5 & 0.513907 \\
\hline & & & & & & 0.7 & 0.499240 \\
\hline
\end{tabular}


Table3: Numerical values of the local Nusselt number $\operatorname{Re}_{b}^{\frac{-1}{n+1}} S h_{x}$ for different values of physical parameters

\begin{tabular}{|c|c|c|c|c|c|c|c|}
\hline $\mathrm{Du}$ & $\mathrm{Sr}$ & $\mathbf{n}$ & $\mathbf{s}$ & Sc & $\mathbf{A}^{*}$ & $\mathrm{~B}^{*}$ & $\operatorname{Re}_{b}{ }^{-1 / n+1} S h_{x}$ \\
\hline 2 & & & & & & & 0.343498 \\
\hline 3 & & & & & & & 0.359337 \\
\hline 4 & & & & & & & 0.378108 \\
\hline 5 & & & & & & & 0.400700 \\
\hline & 0.06 & & & & & & 0.266056 \\
\hline & 0.07 & & & & & & 0.279352 \\
\hline & 0.08 & & & & & & 0.292666 \\
\hline & 0.09 & & & & & & 0.305998 \\
\hline & & 1.1 & & & & & 0.302105 \\
\hline & & 1.4 & & & & & 0.306772 \\
\hline & & 1.7 & & & & & 0.310326 \\
\hline & & 1.9 & & & & & 0.312264 \\
\hline & & & 1.1 & & & & 0.442817 \\
\hline & & & 1.2 & & & & 0.463304 \\
\hline & & & 1.3 & & & & 0.483765 \\
\hline & & & 1.4 & & & & 0.504202 \\
\hline & & & & 0.4 & & & 0.774386 \\
\hline & & & & 0.6 & & & 1.058033 \\
\hline & & & & 0.8 & & & 1.316106 \\
\hline & & & & 1.0 & & & 1.546157 \\
\hline & & & & & 0.2 & & 0.317259 \\
\hline & & & & & 0.4 & & 0.318651 \\
\hline & & & & & 0.6 & & 0.320044 \\
\hline & & & & & 0.8 & & 0.321436 \\
\hline & & & & & & 0.1 & 0.318523 \\
\hline & & & & & & 0.3 & 0.318918 \\
\hline & & & & & & 0.5 & 0.319348 \\
\hline & & & & & & 0.7 & 0.319817 \\
\hline
\end{tabular}

\section{REFERENCES}

1. Abreu, C.R.A., Alfradique, M.F. and Silva, A.T. (2006). Boundary layer flows with Dufour and Soret effects:I:Forced and natural convection, Chemical Engineering Sciencce, Vol. 61, pp. 4282-4289.

2. Alam, M.S. and Rahman, M.M. (2006). Dufour and Soret effects on mixed convection flow past a vertical porous flat plate with variable suction, Nonlinear Analysis: Modeling and Control, Vol. 11, pp. 3-12.

3. Alavala, CHENNAKESAVA R. "Effect of Temperature, Strain Rate and Coefficient of Friction on Deep Drawing Process of 6061 Aluminum Alloy." International Journal of Mechanical Engineering 5.6 (2016): 11-24.

4. Alavala, CHENNAKESAVA R. "FEM analysis of single point incremental forming process and validation with grid-based experimental deformation analysis." International Journal of Mechanical Engineering 5.5 (2016): 1-6.

5. Avinash, K., Sandeep, N. and Makinde, O.D. (2017). Non-uniform heat source/sink effect on liquid film flow of Jeffrey nanofluid over a stretching sheet, Diffusion Foundations, Vol. 11, pp. 72-83.

6. Awais, M., Malik, M.Y., Bilal, S., Salahuddin, T. and Arif, H. (2017). Magneto hydrodynamic (MHD) flow of Sisko fluid near the axisymmetric stagnation point towards a stretching cylinder, Results in Physics, Vol. 7, pp. 49-56.

7. Bhukta, D., Dash, G.C., Mishra S.R. and Baag S. (2017). Dissipation effect on MHD mixed convection flow over a stretching 
sheet through porous medium with non-uniform heat source/sink, Ain Shams Engineering Journal, Vol. 8, pp. 353-361.

8. Bhuvaneswari, M., Sivasankaran, S. and Ferdows, M. (2009). Lie group analysis of natural convection heat and mass transfer in an inclined surface with chemical reaction, Non Linear Analysis: Hybrid Systems, Vol. 3, pp. 536-542.

9. Chhabra, R.P. and Richardson J.F. (2008). Non-Newtonian flow and applied rheology, $2^{\text {nd }}$ Edition, Butter worth-Heinemann, Oxford (2008).

10. Cross, M.M. (1965). Rheology of non-Newtonian fluids: a new flow equation for pseudoplastic systems, Journal of Colloid Science, Vol. 20, pp. 417-437.

11. Darji, R. M. and Timol, M.G. (2014). Similarity analysis for unsteady natural convective boundary layer flow of Sisko fluid, International Journal of Advances in Applied Mathematics and Mechanics, Vol. 1, pp. 22-36.

12. Hayat, T., Asad, S. and Alsaedi, A. (2017). Non-uniform heat source/sink and thermal radiation effects on the stretched flow of cylinder in a thermally stratified medium, Journal of Applied Fluid Mechanics, Vol. 10, pp. 915-924.

13. Hayat, T., Asad, S., Qasim, M. and Hendi, A.A. (2012). Boundary layer flow of a Jeffrey fluid with convective boundary conditions, International Journal for Numerical Methods in Fluids, Vol. 69, pp. 1350-1362.

14. Hayat, T., Mustafa, M. and Pop, I. (2010). Heat and mass transfer for Soret and Dufour's effect on mixed convection boundary layer flow over a stretching vertical surface in a porous medium filled with a viscoelastic fluid, Communications in Nonlinear Science and Numerical Simulation, Vol. 15, pp. 1183-1196.

15. Khan M., Abbas Q. and Duru K. (2010). Magnetohydrodynamic flow of a Sisko fluid in annular pipe: A numerical study, International Journal for Numerical Methods in Fluids, Vol. 62, pp. 1169-1180.

16. Khan, M., Malik, R., Munir, A. and Khan W. A. (2015). Flow and heat transfer to Sisko nanofluid over a nonlinear stretching sheet, PLOS ONE, Vol. 10.

17. Mabood, F. and Ibrahim, S.M. (2016). Effects of Soret and Non-Uniform heat source on MHD non-darcian convective flow over a stretching sheet in a dissipative micropolar fluid with radiation, Journal of Applied Fluid Mechanics, Vol. 9, pp. 25032513.

18. Malik, M.Y., Hussain, A., Salahuddin, T. and Awais, M. (2016). Numerical solution of MHD Sisko fluid over a stretching cylinder and heat transfer analysis, International Journal of Numerical Methods for Heat and Fluid flow, Vol. 26, pp. 17871801 .

19. Malik, R., Khan, M., Munir, A. and Khan, W. A. (2014). Flow and heat transfer in Sisko fluid with convective boundary condition, PLOS ONE, Vol. 9.

20. Mehmood, K., Hussain, S. and Sagheer, M. (2016). Mixed convection flow with non-uniform heat source/sink in a doubly stratified magnetonanofluid, AIP Advances, Vol. 6.

21. Moallemi, N., Shafieenejad, I. and Novinzadeh, A. B. (2011). Exact solutions for flow of a Sisko fluid in pipe, Iranian Mathematical Society, Vol. 37, pp. 49-60.

22. Monica, M. and Sucharitha, J. (2017). Effects of nonlinear thermal radiation, non-uniform heat source/sink on MHD stagnation point flow of a Casson fluid over a nonlinear stretching sheet with slip conditions, Journal of Nanofluids, Vol. 6, pp. 692-701.

23. Munir, A., Shahzad, A. and Khan, M. (2014). Forced convective heat transfer in boundary layer flow of Sisko fluid over a nonlinear stretching sheet, PLOS ONE, Vol. 9. 
24. Mythili, D., Sivaraj, R., Rashidi, M.M. and Yang, Z. (2015). Casson fluid flow over a vertical cone with non-uniform heat source/sink and high order chemical reaction, Journal of Naval Architecture and Marine Engineering, Vol. 15, pp. 125-136.

25. Nadeem, S. and Akbar, N. S. (2010). Peristaltic flow of Sisko fluid in a uniform inclined tube, Acta Mechanica Sinica, Vol. 26, pp. 675-683.

26. Pal, D. (2011). Combined effects of non-uniform heat source/sink and thermal radiation on heat transfer over an unsteady stretching permeable surface, Communications in Nonlinear Science and Numerical Simulation, Vol. 16, pp. 1890-1904.

27. Pal, D. (2015). Unsteady convective boundary layer flow and heat transfer over a stretching surface with non-uniform heat source/sink and thermal radiation, International Journal for Computational Methods in Engineering Science and Mechanics, Vol. 16, pp. 170-181.

28. Pushpalatha, K., Reddy, J. V. R., Sugunamma, V. and Sandeep, N. (2017) Numerical study of chemically reacting unsteady Casson fluid flow past a stretching surface with cross diffusion and thermal radiation, De Gruyter Open, Vol.7, pp. 69-76.

29. Raju, C.S.K. and Sandeep, N. (2016), Heat and mass transfer in MHD non-Newtonian bio-convection flow over a rotating cone/plate with cross diffusion, Journal of Molecular Liquids, Vol. 215, pp.115- 126.

30. Raju, C.S.K., Sandeep, N. and JayachandraBabu, M. (2016).Effects of non-uniform heat source/sink and chemical reaction on unsteady MHD nanofluid flow over a permeable stretching surface, Advanced Science, Engineering and Medicine, Vol. 8, pp. 165-174.

31. Reddy, J. V. R., Sugunamma, V and Sandeep, N. (2016). Crosss diffusion effects on MHD flow over three different geometries with Cattaneo-Christov heat flux, Journal of Molecular Liquids, Vol. 223, pp. 1234-1241.

32. Reddy, J. V. R., Sugunamma, V., Sandeep, N. and Raju, C. S. K. (2017). Chemically reacting MHD dusty nanofluid flow over a vertical cone with non-uniform heat source/sink, Walailak Journal of Science and Technology, Vol. 14, pp. 141-156.

33. Siddiqui, A.M., Ansari, A.R., Ahmad, A. and Ahmad, N. (2009). On Taylor's scraping problem and flow of a Sisko fluid, Mathematical Modelling and Analysis, Vol. 14, pp. 515-529.

34. Sisko, A.W. (1958). The flow of lubricating greases, Industrial and Engineering Chemistry, Vol. 50, pp. 1789 - 1792.

35. Sai, P. Srinivasa, et al. "Chemical reaction and radiation effects on MHD flow over an exponentially stretching sheet with viscous dissipation and heat generation." Int. J. Math. Comput. Appl. Res 5.3 (2015): 3548 (2015).

36. SEKHAR, KV CHANDRA, and V. Manjula. "Heat and mass transfer analysis of steady mhd free convective fluid flow past an inclined stretching porous sheet with viscous dissipation and radiation." (2018).

37. Tsai, R., Huang, K. H., Huang, J.S. (2008). Flow and heat transfer over an unsteady stretching surface with non-uniform heat source, International Communications in Heat and Mass Transfer, Vol. 35, pp. 1340-1343. 

